\title{
Up-down asymmetries of charmed baryon three-body decays
}

\author{
Jian-Yong Cen ${ }^{1}$, Chao-Qiang Geng ${ }^{1,2,3, a}$, Chia-Wei Liu ${ }^{2}$, Tien-Hsueh Tsai ${ }^{2}$ \\ ${ }^{1}$ School of Physics and Information Engineering, Shanxi Normal University, Linfen 041004, China \\ 2 Department of Physics, National Tsing Hua University, Hsinchu 300, Taiwan \\ ${ }^{3}$ Physics Division, National Center for Theoretical Sciences, Hsinchu 300, Taiwan
}

Received: 2 October 2019 / Accepted: 6 November 2019 / Published online: 19 November 2019

(C) The Author(s) 2019

\begin{abstract}
We study the up-down asymmetries in the threebody anti-triplet charmed baryon decays of $\mathbf{B}_{\mathbf{c}} \rightarrow \mathbf{B}_{\mathbf{n}} M M^{\prime}$ with the $S U(3)_{f}$ flavor symmetry, where $\mathbf{B}_{\mathbf{c}}$ presents the anti-triplet charmed baryon of $\left(\Xi_{c}^{0},-\Xi_{c}^{+}, \Lambda_{c}^{+}\right)$, while $\mathbf{B}_{\mathbf{n}}$ and $M^{\left({ }^{\prime}\right)}$ denote octet baryon and meson states, respectively. By assuming the s-wave meson-pairs to be the dominant constituents in final state configurations, we can write the spin-dependent decay amplitude into parity-conserving and parity-violating parts, parametrized by 6 real parameters under $S U(3)_{f}$, respectively. Fitting these parameters by 16 experimental data points with the minimum $\chi^{2}$ method, we obtain that $\chi^{2} /$ d.o. $f=2.4$. With the fitted parameters, we evaluate the up-down asymmetries along with the decay branching ratios of $\mathbf{B}_{\mathbf{c}} \rightarrow \mathbf{B}_{\mathbf{n}} M M^{\prime}$. Some of these up-down asymmetries are accessible to the experiments at BESIII, BELLE-II and LHCb.
\end{abstract}

\section{Introduction}

The three-body charmed baryon decays of $\mathbf{B}_{\mathbf{c}} \rightarrow \mathbf{B}_{\mathbf{n}} M M^{\prime}$ have been recently searched by the experimental Collaborations of BELLE, BESIII and LHCb, where $\mathbf{B}_{\mathbf{c}} \equiv$ $\left(\Xi_{c}^{0},-\Xi_{c}^{+}, \Lambda_{c}^{+}\right)$denotes the charmed baryon anti-triplet, while $\mathbf{B}_{\mathbf{n}}$ and $M^{\left({ }^{\prime}\right)}$ correspond to the baryon and meson octets, respectively. In particular, the golden mode of $\Lambda_{c}^{+} \rightarrow$ $p K^{-} \pi^{+}$has been measured with high precision by BELLE and BESIII [1,2], which can improve the accuracies of other $\Lambda_{c}^{+}$decays which are usually given by the rates relative to it [3]. Since the decay branching ratio is a spin-averaged observable, it loses the ability to probe the polarization property of the parent or daughter baryon in a baryonic decay. On the other hand, several $\mathrm{P}$ and $\mathrm{CP}$ or $\mathrm{T}$ violating spin correlations can be constructed due to the rich spin structures in the three-body baryonic decays. In order to understand the full dynamics of decay processes, a systematic study, which has

a e-mail: geng@phys.nthu.edu.tw been done in two-body modes [4], on both branching ratios and up-down asymmetries, also known as daughter-baryon spin polarizations, in the three-body charmed baryon modes is necessary. In this work, we only concentrate on the $\mathrm{P}$ violating up-down asymmetries in $\mathbf{B}_{\mathbf{c}} \rightarrow \mathbf{B}_{\mathbf{n}} M M^{\prime}$, which have not been examined both experimentally and theoretically in the literature yet.

However, it is known that the investigation into the charm baryon decays has always been difficult. The main reason for this is that the scale of the charm quark mass is too large for the flavor $S U(4)_{f}$ symmetry, so that the heavy to light quark transitions $(c \rightarrow q)$ in charmed decays cannot be easily calculated. Moreover, the factorization method fails in these decays [5], in addition to that the three-body processes are much more complicated than the two-body ones. The alternative approaches for the charmed hadron decays have been shown in Refs. [6-13], where the non-factorizable effects are taken into account. On the other hand, the $S U(3)_{f}$ symmetry has been tested as a useful tool in the charmed hadronic decays $[4,14-28]$.

In order to study $\mathbf{B}_{\mathbf{c}} \rightarrow \mathbf{B}_{\mathbf{n}} M M^{\prime}$, we assume that the final state configurations of the meson-pairs are dominated by the s-wave ones, so that the three-body system can be treated as an effective two-body one with an off-shell scalar meson [25] to analogize the three-body semileptonic decays of charmed baryons [27]. Similar to the discussions on the up-down asymmetries in the two-body decays of $\mathbf{B}_{\mathbf{c}} \rightarrow \mathbf{B}_{\mathbf{n}} M$ in Ref. [4], we define the spin dependent decay amplitudes in terms of parity-conserving and violating parts under $S U(3)_{f}$, respectively, resulting in 12 real parameters to be fitted with 16 available data points. In our study, we also consider the the kinematic correction factors as in Ref. [25] to keep the triangle relations derived in Refs. [18,25,29], but break those by the U-spin symmetry [30] due to the large differences of hadron masses.

This paper is organized as follows. In Sect. 2, we give the formalism and display the explicit amplitudes for the 
three-body charmed baryon decays of $\mathbf{B}_{\mathbf{c}} \rightarrow \mathbf{B}_{\mathbf{n}} M M^{\prime}$ under the $S U(3)_{f}$ symmetry. In Sect. 3, we present our numerical results and discussions. Our conclusions are shown in Sect. 4.

\section{Formalism}

In order to study the up-down asymmetries of the three-body $\mathbf{B}_{\mathbf{c}} \rightarrow \mathbf{B}_{\mathbf{n}} M M^{\prime}$ non-leptonic decays, we start with the charm quark decays of $c \rightarrow s \bar{u} d, c \rightarrow u \bar{d} d(u \bar{s} s)$ and $c \rightarrow d \bar{u} s$ at tree-level with the effective Hamiltonian, given by [31]

$$
\begin{aligned}
\mathcal{H}_{\text {eff }}= & \sum_{i=-,+} \frac{G_{F}}{\sqrt{2}} c_{i}\left[V_{c s} V_{u d}^{*} O_{i}^{d s}\right. \\
& \left.+V_{c d} V_{u d}^{*} O_{i}^{q q}+V_{c d} V_{u s}^{*} O_{i}^{s d}\right],
\end{aligned}
$$

with

$$
\begin{aligned}
O_{ \pm}^{q_{1} q_{2}} & \left.\left.\left.=\frac{1}{2}\left[\left(\bar{u} q_{1}\right)_{V-A}\left(\bar{q}_{2} c\right)\right)_{V-A} \pm\left(\bar{q}_{2} q_{1}\right)\right)_{V-A}(\bar{u} c)\right)_{V-A}\right] \\
O_{ \pm}^{q q} & =O_{ \pm}^{d d}-O_{ \pm}^{s s}
\end{aligned}
$$

where $G_{F}$ is the Fermi constant, $c_{ \pm}$represent the Wilson coefficients and $\left(V_{c s} V_{u d}, V_{c d} V_{u d}, V_{c d} V_{u s}\right)=c_{c}^{2}\left(1,-t_{c},-t_{c}^{2}\right)$ correspond to the CKM matrix elements with $c_{c}=\cos \theta_{c}$, $t_{c}=\sin \theta_{c} / \cos \theta_{c}$ and $\theta_{c}$ the Cabibbo angle. Here, the relation of $V_{c s} V_{u s}=-V_{c d} V_{u d}$ has been used to combine the $c \rightarrow u d \bar{d}(u s \bar{s})$ transitions, $\left(\bar{q}_{1} q_{2}\right)_{V-A}\left(\bar{q}_{3} c\right)_{V-A} \equiv$ $\bar{q}_{1} \gamma_{\mu}\left(1-\gamma_{5}\right) q_{2} \bar{q}_{3} \gamma^{\mu}\left(1-\gamma_{5}\right) c$ in $O_{ \pm}^{q_{1} q_{2}}$ and $O_{ \pm}^{q q}$ are the fourquark operators, and the decays of $O_{ \pm}^{d s}, O_{ \pm}^{q q}$ and $O_{ \pm}^{s d}$ are so-called Cabibbo-favored (CF), singly Cabibbo-suppressed (SCS), and doubly Cabibbo-suppressed (DCS) processes, respectively.

In Eq. (2), the flavor structure of the four quark operator $\left(\bar{q}_{1} q_{2}\right)_{V-A}\left(\bar{q}_{3} c\right)_{V-A}$ can be rewritten as $\left(\bar{q}^{i} q_{k} \bar{q}^{j}\right) c$ with $q_{i}=$ $(u, d, s)$, which is the triplet of 3 under the $S U(3)_{f}$ symmetry, where the Dirac and Lorentz indices are suppressed for simplicity. Since $\left(\bar{q}^{i} q_{k} \bar{q}^{j}\right) c$ can be decomposed as the irreducible representations of $(\overline{3} \times 3 \times \overline{3}) c=\left(\overline{3}+\overline{3}^{\prime}+6+\overline{15}\right) c$, one can derive that $[14,15]$

$$
\begin{aligned}
& O_{+(-)}^{d s} \simeq \mathcal{O}_{\frac{d s}{15(6)}}=\frac{1}{2}(\bar{u} d \bar{s} \pm \bar{s} d \bar{u}) c, \\
& O_{-(+)}^{q q} \simeq \mathcal{O}_{\frac{q q}{15(6)}}^{q q}=\frac{1}{2}(\bar{u} d \bar{d} \pm \bar{d} d \bar{u}) c-\frac{1}{2}(\bar{u} s \bar{s} \pm \bar{s} s \bar{u}) c, \\
& O_{-(+)}^{s d} \simeq \mathcal{O}_{\frac{s d}{15(6)}}=\frac{1}{2}(\bar{u} s \bar{d} \pm \bar{d} s \bar{u}) c .
\end{aligned}
$$

Consequently, the effective Hamiltonian in Eq. (1) has the expression under the $S U(3)_{f}$ symmetry, given by $[19,20,22-$ 24]

$\mathcal{H}_{e f f}=\frac{G_{F}}{\sqrt{2}}\left[c_{-} \frac{\epsilon^{i j l}}{2} H(6)_{l k}+c_{+} H(\overline{15})_{k}^{i j}\right] c$, where $(i, j, k)$ are the flavor indices, while $H(6)$ and $H(\overline{15})$ are symmetric and anti-symmetric flavor tensors of $\mathcal{O}_{6,15}^{\left(q_{1} q_{2}, q q\right)}$ in Eq. (3), with their non-zero entries given by $[14,15]$

$$
\begin{aligned}
H(6)_{i j} & =c_{s}^{2}\left(\begin{array}{ccc}
0 & 0 & 0 \\
0 & 2 & 2 t_{c} \\
0 & 2 t_{c} & 2 t_{c}^{2}
\end{array}\right), \\
H(\overline{15})_{k}^{i j} & =c_{s}^{2}\left(\left(\begin{array}{lll}
0 & 0 & 0 \\
0 & 0 & 0 \\
0 & 0 & 0
\end{array}\right),\left(\begin{array}{ccc}
0 & -t_{c} & 1 \\
-t_{c} & 0 & 0 \\
1 & 0 & 0
\end{array}\right),\left(\begin{array}{ccc}
0 & -t_{c}^{2} & t_{c} \\
-t_{c}^{2} & 0 & 0 \\
t_{c} & 0 & 0
\end{array}\right)\right),
\end{aligned}
$$

respectively. The three lowest-lying charmed baryon states of $\mathbf{B}_{c}$ form an anti-triplet of $\overline{3}$ to consist of $(d s-s d) c,(u s-s u) c$ and $(u d-d u) c$, and $\mathbf{B}_{n}$ and $M$ belong to octet (8) states of the baryon and meson, which are written as

$\mathbf{B}_{c}=\left(\Xi_{c}^{0},-\Xi_{c}^{+}, \Lambda_{c}^{+}\right)$,

$\mathbf{B}_{n}=\left(\begin{array}{ccc}\frac{1}{\sqrt{6}} \Lambda^{0}+\frac{1}{\sqrt{2}} \Sigma^{0} & \Sigma^{-} & \Xi^{-} \\ \Sigma^{+} & \frac{1}{\sqrt{6}} \Lambda^{0}-\frac{1}{\sqrt{2}} \Sigma^{0} & \Xi^{0} \\ p & n & -\sqrt{\frac{2}{3}} \Lambda^{0}\end{array}\right)$,

$M=\left(\begin{array}{ccc}\frac{1}{\sqrt{2}} \pi^{0}+\frac{1}{\sqrt{6}} \eta & \pi^{-} & K^{-} \\ \pi^{+} & -\frac{1}{\sqrt{2}} \pi^{0}+\frac{1}{\sqrt{6}} \eta & \bar{K}^{0} \\ K^{+} & K^{0} & -\sqrt{\frac{2}{3}} \eta\end{array}\right)$,

respectively.

In the NDR scheme, the Wilson coefficients of $\left(c_{-}, c_{+}\right)$ are found to be $(1.78,0.76)$ at the scale of $\mu=1 \mathrm{GeV}$ [32], resulting in that the amplitudes associated with $H(\overline{15})$ are suppressed due to the value of $\left(c_{-} / c_{+}\right)^{2} \simeq 5.5$. In addition, the nonfactorizable contributions to the decays from $H(\overline{15})$ are zero due to the vanishing baryonic transition matrix elements from $\overline{\mathbf{1 5}}$ [13], while the factorizable are found to be small in most of the modes [24]. For the three-day decay of $\mathbf{B}_{\mathbf{c}} \rightarrow \mathbf{B}_{\mathbf{n}} M M^{\prime}$, in this study we only consider the s-wave meson-pair in the final-state configuration, regarded as a offshell scalar particle inspired. As a result, the spin dependent decay amplitude can be simply written as

$$
\begin{aligned}
\mathcal{M}\left(\mathbf{B}_{\mathbf{c}} \rightarrow \mathbf{B}_{\mathbf{n}} M M^{\prime}\right) & =\left\langle\mathbf{B}_{\mathbf{n}} M M^{\prime}\left|\mathcal{H}_{e f f}\right| \mathbf{B}_{\mathbf{c}}\right\rangle \\
& =i \bar{u}_{\mathbf{B}_{\mathbf{n}}}\left(A-B \gamma_{5}\right) u_{\mathbf{B}_{\mathbf{c}}}
\end{aligned}
$$

where $u_{\mathbf{B}_{\mathbf{c}, \mathbf{n}}}$ are Dirac spinors of baryons, while $A$ and $B$ correspond to the parity conserving and parity violating processes, which can be represented by $S U$ (3) irreducible amplitudes, given by

$$
\begin{aligned}
& A\left(\mathbf{B}_{\mathbf{c}} \rightarrow \mathbf{B}_{n} M M^{\prime}\right) \\
& =a_{1}\left(\overline{\mathbf{B}}_{n}\right)_{i}^{k}(M)_{l}^{m}(M)_{m}^{l} H(6)_{j k} T^{i j} \\
& \quad+a_{2}\left(\overline{\mathbf{B}}_{n}\right)_{i}^{k}(M)_{j}^{m}(M)_{m}^{l} H(6)_{k l} T^{i j} \\
& \quad+a_{3}\left(\overline{\mathbf{B}}_{n}\right)_{i}^{k}(M)_{k}^{m}(M)_{m}^{l} H(6)_{j l} T^{i j} \\
& \quad+a_{4}\left(\overline{\mathbf{B}}_{n}\right)_{i}^{k}(M)_{j}^{l}(M)_{k}^{m} H(6)_{l m} T^{i j}
\end{aligned}
$$


Table 1 A-amplitudes of $\Lambda_{c}^{+} \rightarrow \mathbf{B}_{\mathbf{n}} M M^{\prime}$

\begin{tabular}{|c|c|c|c|c|c|}
\hline CF mode & $A$ & CS mode & $A t_{c}^{-1}$ & DCS mode & $A t_{c}^{-2}$ \\
\hline$\Sigma^{+} \pi^{0} \pi^{0}$ & $4 a_{1}+2 a_{2}+2 a_{3}+2 a_{4}-2 a_{5}$ & $\Sigma^{+} \pi^{0} K^{0}$ & $\sqrt{2} a_{2}+\sqrt{2} a_{3}+2 \sqrt{2} a_{4}$ & $\Sigma^{+} K^{0} K^{0}$ & $4 a_{4}$ \\
\hline$\Sigma^{+} \pi^{+} \pi^{-}$ & $4 a_{1}+2 a_{2}+2 a_{3}-2 a_{5}-2 a_{6}$ & $\Sigma^{+} \pi^{-} K^{+}$ & $-2 a_{2}-2 a_{3}+2 a_{6}$ & $\Sigma^{0} K^{0} K^{+}$ & $2 \sqrt{2} a_{4}$ \\
\hline$\Sigma^{+} K^{0} \bar{K}^{0}$ & $4 a_{1}+2 a_{2}+2 a_{3}$ & $\Sigma^{+} K^{0} \eta^{0}$ & $\frac{\sqrt{6} a_{2}}{3}+\frac{\sqrt{6} a_{3}}{3}-\frac{2 \sqrt{6} a_{4}}{3}$ & $\Sigma^{-} K^{+} K^{+}$ & $-4 a_{4}$ \\
\hline$\Sigma^{+} K^{+} K^{-}$ & $4 a_{1}-2 a_{5}$ & $\Sigma^{0} \pi^{+} K^{0}$ & $-\sqrt{2} a_{2}-\sqrt{2} a_{3}-2 \sqrt{2} a_{4}$ & $p \pi^{0} K^{0}$ & $-\sqrt{2} a_{2}$ \\
\hline$\Sigma^{+} \eta^{0} \eta^{0}$ & $4 a_{1}+\frac{2 a_{2}}{3}+\frac{2 a_{3}}{3}+\frac{2 a_{4}}{3}-\frac{2 a_{5}}{3}$ & $\Sigma^{0} K^{+} \eta^{0}$ & $\frac{\sqrt{3} a_{2}}{3}+\frac{\sqrt{3} a_{3}}{3}-\frac{2 \sqrt{3} a_{4}}{3}$ & $p \pi^{-} K^{+}$ & $2 a_{2}$ \\
\hline$\Sigma^{0} \pi^{0} \pi^{+}$ & $-2 a_{4}-2 a_{6}$ & $\Sigma^{-} \pi^{+} K^{+}$ & $4 a_{4}+2 a_{6}$ & $p K^{0} \eta^{0}$ & $-\frac{\sqrt{6} a_{2}}{3}-\frac{2 \sqrt{6} a_{4}}{3}$ \\
\hline$\Sigma^{0} K^{+} \bar{K}^{0}$ & $\sqrt{2} a_{2}+\sqrt{2} a_{3}+\sqrt{2} a_{5}$ & $p \pi^{0} \pi^{0}$ & $-4 a_{1}-2 a_{2}+2 a_{5}$ & $n \pi^{0} K^{+}$ & $-\sqrt{2} a_{2}$ \\
\hline$\Sigma^{-} \pi^{+} \pi^{+}$ & $-4 a_{4}-4 a_{6}$ & $p \pi^{0} \eta^{0}$ & $\frac{2 \sqrt{3} a_{2}}{3}-\frac{2 \sqrt{3} a_{4}}{3}+\frac{2 \sqrt{3} a_{5}}{3}$ & $n \pi^{+} K^{0}$ & $-2 a_{2}$ \\
\hline$\Xi^{0} \pi^{0} K^{+}$ & $-\sqrt{2} a_{5}$ & $p \pi^{+} \pi^{-}$ & $-4 a_{1}-2 a_{2}+2 a_{5}$ & $n K^{+} \eta^{0}$ & $\frac{\sqrt{6} a_{2}}{3}+\frac{2 \sqrt{6} a_{4}}{3}$ \\
\hline$\Xi^{0} \pi^{+} K^{0}$ & $-2 a_{5}-2 a_{6}$ & $p K^{+} K^{-}$ & $-4 a_{1}-2 a_{3}+2 a_{5}+2 a_{6}$ & & \\
\hline$\Xi^{-} \pi^{+} K^{+}$ & $-2 a_{6}$ & $p \eta^{0} \eta^{0}$ & $-4 a_{1}-\frac{2 a_{2}}{3}-\frac{8 a_{3}}{3}+\frac{4 a_{4}}{3}+\frac{2 a_{5}}{3}$ & & \\
\hline$p \pi^{0} \bar{K}^{0}$ & $-\sqrt{2} a_{3}-\sqrt{2} a_{4}$ & $n \pi^{+} \eta^{0}$ & $\frac{2 \sqrt{6} a_{2}}{3}-\frac{2 \sqrt{6} a_{4}}{3}+\frac{2 \sqrt{6} a_{5}}{3}$ & & \\
\hline$p \pi^{+} K^{-}$ & $2 a_{3}-2 a_{6}$ & $n K^{+} \bar{K}^{0}$ & $2 a_{2}+2 a_{4}+2 a_{5}+2 a_{6}$ & & \\
\hline$p \bar{K}^{0} \eta^{0}$ & $-\frac{\sqrt{6} a_{3}}{3}+\frac{\sqrt{6} a_{4}}{3}$ & $\Lambda^{0} \pi^{0} K^{+}$ & $\frac{\sqrt{3} a_{2}}{3}-\frac{\sqrt{3} a_{3}}{3}-\frac{2 \sqrt{3} a_{5}}{3}$ & & \\
\hline$n \pi^{+} \bar{K}^{0}$ & $-2 a_{4}-2 a_{6}$ & $\Lambda^{0} \pi^{+} K^{0}$ & $\frac{\sqrt{6} a_{2}}{3}-\frac{\sqrt{6} a_{3}}{3}-\frac{2 \sqrt{6} a_{5}}{3}$ & & \\
\hline$\Lambda^{0} \pi^{+} \eta^{0}$ & $-\frac{2 a_{2}}{3}+\frac{2 a_{3}}{3}-\frac{2 a_{5}}{3}-2 a_{6}$ & $\Lambda^{0} K^{+} \eta^{0}$ & $-\frac{a_{2}}{3}+\frac{a_{3}}{3}+\frac{2 a_{5}}{3}+2 a_{6}$ & & \\
\hline$\Lambda^{0} K^{+} \bar{K}^{0}$ & $-\frac{\sqrt{6} a_{2}}{3}+\frac{\sqrt{6} a_{3}}{3}-\frac{\sqrt{6} a_{5}}{3}$ & & & & \\
\hline
\end{tabular}

Table 2 A-amplitudes of $\mathbf{\Xi}_{c}^{+} \rightarrow \mathbf{B}_{\mathbf{n}} M M^{\prime}$

\begin{tabular}{|c|c|c|c|c|c|}
\hline $\mathrm{CF}$ mode & $A$ & CS mode & $A t_{c}^{-1}$ & DCS mode & $A t_{c}^{-2}$ \\
\hline$\Sigma^{+} \pi^{0} \bar{K}^{0}$ & $-\sqrt{2} a_{2}-\sqrt{2} a_{4}$ & $\Sigma^{+} \pi^{0} \pi^{0}$ & $-4 a_{1}-2 a_{3}+2 a_{5}$ & $\Sigma^{+} \pi^{0} K^{0}$ & $-\sqrt{2} a_{3}$ \\
\hline$\Sigma^{+} \pi^{+} K^{-}$ & $2 a_{2}$ & $\Sigma^{+} \pi^{0} \eta^{0}$ & $\frac{2 \sqrt{3} a_{3}}{3}-\frac{2 \sqrt{3} a_{4}}{3}+\frac{2 \sqrt{3} a_{5}}{3}$ & $\Sigma^{+} \pi^{-} K^{+}$ & $2 a_{3}-2 a_{6}$ \\
\hline$\Sigma^{+} \bar{K}^{0} \eta^{0}$ & $-\frac{\sqrt{6} a_{2}}{3}+\frac{\sqrt{6} a_{4}}{3}$ & $\Sigma^{+} \pi^{+} \pi^{-}$ & $-4 a_{1}-2 a_{3}+2 a_{5}+2 a_{6}$ & $\Sigma^{+} K^{0} \eta^{0}$ & $-\frac{\sqrt{6} a_{3}}{3}-\frac{2 \sqrt{6} a_{4}}{3}$ \\
\hline$\Sigma^{0} \pi^{+} \bar{K}^{0}$ & $\sqrt{2} a_{4}$ & $\Sigma^{+} K^{+} K^{-}$ & $-4 a_{1}-2 a_{2}+2 a_{5}$ & $\Sigma^{0} \pi^{0} K^{+}$ & $a_{3}-2 a_{6}$ \\
\hline$\Xi^{0} \pi^{0} \pi^{+}$ & $\sqrt{2} a_{4}$ & $\Sigma^{+} \eta^{0} \eta^{0}$ & $-4 a_{1}-\frac{8 a_{2}}{3}-\frac{2 a_{3}}{3}+\frac{4 a_{4}}{3}+\frac{2 a_{5}}{3}$ & $\Sigma^{0} \pi^{+} K^{0}$ & $\sqrt{2} a_{3}$ \\
\hline$\Xi^{0} \pi^{+} \eta^{0}$ & $-\frac{2 \sqrt{6} a_{2}}{3}-\frac{\sqrt{6} a_{4}}{3}$ & $\Sigma^{0} \pi^{0} \pi^{+}$ & $2 a_{6}$ & $\Sigma^{0} K^{+} \eta^{0}$ & $-\frac{\sqrt{3} a_{3}}{3}-\frac{2 \sqrt{3} a_{4}}{3}$ \\
\hline$\Xi^{0} K^{+} \bar{K}^{0}$ & $-2 a_{2}$ & $\Sigma^{0} \pi^{+} \eta^{0}$ & $-\frac{2 \sqrt{3} a_{3}}{3}+\frac{2 \sqrt{3} a_{4}}{3}-\frac{2 \sqrt{3} a_{5}}{3}$ & $\Sigma^{-} \pi^{+} K^{+}$ & $-2 a_{6}$ \\
\hline$\Xi^{-} \pi^{+} \pi^{+}$ & $-4 a_{4}$ & $\Sigma^{0} K^{+} \bar{K}^{0}$ & $-\sqrt{2} a_{3}-\sqrt{2} a_{4}-\sqrt{2} a_{5}$ & $\Xi^{0} K^{0} K^{+}$ & $-2 a_{4}-2 a_{6}$ \\
\hline$p \bar{K}^{0} \bar{K}^{0}$ & $4 a_{4}$ & $\Sigma^{-} \pi^{+} \pi^{+}$ & $4 a_{6}$ & $\Xi^{-} K^{+} K^{+}$ & $-4 a_{4}-4 a_{6}$ \\
\hline \multirow[t]{10}{*}{$\Lambda^{0} \pi^{+} \bar{K}^{0}$} & $\sqrt{6} a_{4}$ & $\Xi^{0} \pi^{0} K^{+}$ & $\sqrt{2} a_{2}-\sqrt{2} a_{4}+\sqrt{2} a_{5}$ & $p \pi^{0} \pi^{0}$ & $4 a_{1}-2 a_{5}$ \\
\hline & & $\Xi^{0} \pi^{+} K^{0}$ & $2 a_{2}+2 a_{4}+2 a_{5}+2 a_{6}$ & $p \pi^{0} \eta^{0}$ & $-\frac{2 \sqrt{3} a_{5}}{3}$ \\
\hline & & $\Xi^{0} K^{+} \eta^{0}$ & $-\frac{\sqrt{6} a_{2}}{3}+\frac{\sqrt{6} a_{4}}{3}-\frac{\sqrt{6} a_{5}}{3}$ & $p \pi^{+} \pi^{-}$ & $4 a_{1}-2 a_{5}$ \\
\hline & & $\Xi^{-} \pi^{+} K^{+}$ & $4 a_{4}+2 a_{6}$ & $p K^{0} \bar{K}^{0}$ & $4 a_{1}+2 a_{2}+2 a_{3}$ \\
\hline & & $p \pi^{0} \bar{K}^{0}$ & $\sqrt{2} a_{2}+\sqrt{2} a_{3}$ & $p K^{+} K^{-}$ & $4 a_{1}+2 a_{2}+2 a_{3}-2 a_{5}-2 a_{6}$ \\
\hline & & $p \pi^{+} K^{-}$ & $-2 a_{2}-2 a_{3}+2 a_{6}$ & $p \eta^{0} \eta^{0}$ & $4 a_{1}+\frac{8 a_{2}}{3}+\frac{8 a_{3}}{3}+\frac{8 a_{4}}{3}-\frac{2 a_{5}}{3}$ \\
\hline & & $p \bar{K}^{0} \eta^{0}$ & $\frac{\sqrt{6} a_{2}}{3}+\frac{\sqrt{6} a_{3}}{3}+\frac{4 \sqrt{6} a_{4}}{3}$ & $n \pi^{+} \eta^{0}$ & $-\frac{2 \sqrt{6} a_{5}}{3}$ \\
\hline & & $n \pi^{+} \bar{K}^{0}$ & $2 a_{6}$ & $n K^{+} \bar{K}^{0}$ & $-2 a_{5}-2 a_{6}$ \\
\hline & & $\Lambda^{0} \pi^{+} \eta^{0}$ & $-\frac{4 a_{2}}{3}-\frac{2 a_{3}}{3}+2 a_{4}+\frac{2 a_{5}}{3}+2 a_{6}$ & $\Lambda^{0} \pi^{0} K^{+}$ & $\frac{2 \sqrt{3} a_{2}}{3}+\frac{\sqrt{3} a_{3}}{3}+\frac{2 \sqrt{3} a_{5}}{3}$ \\
\hline & & $\Lambda^{0} K^{+} \bar{K}^{0}$ & $-\frac{2 \sqrt{6} a_{2}}{3}-\frac{\sqrt{6} a_{3}}{3}-\sqrt{6} a_{4}+\frac{\sqrt{6} a_{5}}{3}$ & $\Lambda^{0} \pi^{+} K^{0}$ & $\frac{2 \sqrt{6} a_{2}}{3}+\frac{\sqrt{6} a_{3}}{3}+\frac{2 \sqrt{6} a_{5}}{3}$ \\
\hline
\end{tabular}


Table 3 A-amplitudes of $\Xi_{c}^{0} \rightarrow \mathbf{B}_{\mathbf{n}} M M^{\prime}$

\begin{tabular}{|c|c|c|c|c|c|}
\hline CF mode & $A$ & CS mode & $A t_{c}^{-1}$ & DCS mode & $A t_{c}^{-2}$ \\
\hline$\Sigma^{+} \pi^{0} K^{-}$ & $\sqrt{2} a_{5}$ & $\Sigma^{+} \pi^{0} \pi^{-}$ & $-\sqrt{2} a_{6}$ & $\Sigma^{+} \pi^{-} K^{0}$ & $-2 a_{6}$ \\
\hline$\Sigma^{+} \pi^{-} \bar{K}^{0}$ & $2 a_{5}+2 a_{6}$ & $\Sigma^{+} \pi^{-} \eta^{0}$ & $\frac{2 \sqrt{6} a_{5}}{3}+\sqrt{6} a_{6}$ & $\Sigma^{0} \pi^{0} K^{0}$ & $a_{3}-2 a_{6}$ \\
\hline$\Sigma^{+} K^{-} \eta^{0}$ & $-\frac{\sqrt{6} a_{5}}{3}$ & $\Sigma^{+} K^{0} K^{-}$ & $2 a_{5}$ & $\Sigma^{0} \pi^{-} K^{+}$ & $-\sqrt{2} a_{3}$ \\
\hline$\Sigma^{0} \pi^{0} \bar{K}^{0}$ & $a_{2}+a_{4}+a_{5}+2 a_{6}$ & $\Sigma^{0} \pi^{0} \pi^{0}$ & $2 \sqrt{2} a_{1}+\sqrt{2} a_{3}-\sqrt{2} a_{5}-2 \sqrt{2} a_{6}$ & $\Sigma^{0} K^{0} \eta^{0}$ & $\frac{\sqrt{3} a_{3}}{3}+\frac{2 \sqrt{3} a_{4}}{3}$ \\
\hline$\Sigma^{0} \pi^{+} K^{-}$ & $-\sqrt{2} a_{2}-\sqrt{2} a_{5}$ & $\Sigma^{0} \pi^{0} \eta^{0}$ & $-\frac{\sqrt{6} a_{3}}{3}+\frac{\sqrt{6} a_{4}}{3}+\frac{\sqrt{6} a_{5}}{3}+\sqrt{6} a_{6}$ & $\Sigma^{-} \pi^{0} K^{+}$ & $\sqrt{2} a_{3}$ \\
\hline$\Sigma^{0} \bar{K}^{0} \eta^{0}$ & $\frac{\sqrt{3} a_{2}}{3}-\frac{\sqrt{3} a_{4}}{3}+\frac{\sqrt{3} a_{5}}{3}$ & $\Sigma^{0} \pi^{+} \pi^{-}$ & $2 \sqrt{2} a_{1}+\sqrt{2} a_{3}-\sqrt{2} a_{5}$ & $\Sigma^{-} \pi^{+} K^{0}$ & $2 a_{3}-2 a_{6}$ \\
\hline$\Sigma^{-} \pi^{+} \bar{K}^{0}$ & $2 a_{4}+2 a_{6}$ & $\Sigma^{0} K^{0} \bar{K}^{0}$ & $\sqrt{2}\left(2 a_{1}+a_{2}+a_{3}+a_{4}-a_{5}\right)$ & $\Sigma^{-} K^{+} \eta^{0}$ & $-\frac{\sqrt{6} a_{3}}{3}-\frac{2 \sqrt{6} a_{4}}{3}$ \\
\hline$\Xi^{0} \pi^{0} \eta^{0}$ & $\frac{2 \sqrt{3} a_{2}}{3}+\frac{2 \sqrt{3} a_{3}}{3}+\frac{2 \sqrt{3} a_{4}}{3}$ & $\Sigma^{0} K^{+} K^{-}$ & $2 \sqrt{2} a_{1}+\sqrt{2} a_{2}$ & $\Xi^{0} K^{0} K^{0}$ & $-4 a_{4}-4 a_{6}$ \\
\hline$\Xi^{0} \pi^{+} \pi^{-}$ & $-4 a_{1}-2 a_{2}-2 a_{3}$ & $\Sigma^{0} \eta^{0} \eta^{0}$ & $\sqrt{2}\left(2 a_{1}+\frac{4 a_{2}}{3}+\frac{a_{3}}{3}-\frac{2 a_{4}}{3}-\frac{a_{5}}{3}\right)$ & $\Xi^{-} K^{0} K^{+}$ & $-2 a_{4}-2 a_{6}$ \\
\hline$\Xi^{0} K^{0} \bar{K}^{0}$ & $-2\left(2 a_{1}+a_{2}+a_{3}\right.$ & $\Sigma^{-} \pi^{0} \pi^{+}$ & $-\sqrt{2} a_{6}$ & $p \pi^{-} \eta^{0}$ & $-\frac{2 \sqrt{6} a_{5}}{3}$ \\
\hline & $\left.-a_{5}-a_{6}\right)$ & $\Sigma^{-} \pi^{+} \eta^{0}$ & $-\frac{2 \sqrt{6} a_{3}}{3}+\frac{2 \sqrt{6} a_{4}}{3}+\sqrt{6} a_{6}$ & $p K^{0} K^{-}$ & $-2 a_{5}-2 a_{6}$ \\
\hline$\Xi^{0} K^{+} K^{-}$ & $-4 a_{1}+2 a_{5}$ & $\Sigma^{-} K^{+} \bar{K}^{0}$ & $-2 a_{3}-2 a_{4}$ & $n \pi^{0} \pi^{0}$ & $4 a_{1}-2 a_{5}$ \\
\hline$\Xi^{0} \eta^{0} \eta^{0}$ & $\begin{array}{l}-2\left(2 a_{1}+\frac{a_{2}}{3}+\frac{a_{3}}{3}\right. \\
\left.+\frac{a_{4}}{3}-\frac{4 a_{5}}{3}\right)\end{array}$ & $\begin{array}{l}\Xi^{0} \pi^{-} K^{+} \\
\Xi^{0} K^{0} \eta^{0}\end{array}$ & $\begin{array}{l}2 a_{2}+2 a_{3}+2 a_{5} \\
\sqrt{6}\left(-\frac{a_{2}}{3}-\frac{a_{3}}{3}+\frac{2 a_{4}}{3}-\frac{a_{5}}{3}+a_{6}\right)\end{array}$ & $\begin{array}{l}n \pi^{0} \eta^{0} \\
n \pi^{+} \pi^{-}\end{array}$ & $\begin{array}{l}\frac{2 \sqrt{3} a_{5}}{3} \\
4 a_{1}-2 a_{5}\end{array}$ \\
\hline$\Xi^{-} \pi^{0} \pi^{+}$ & $\sqrt{2} a_{4}$ & $\Xi^{-} \pi^{0} K^{+}$ & $\sqrt{2} a_{3}-\sqrt{2} a_{4}-\sqrt{2} a_{6}$ & $n K^{0} \bar{K}^{0}$ & $2\left(2 a_{1}+a_{2}+a_{3}\right.$ \\
\hline$\Xi^{-} \pi^{+} \eta^{0}$ & $-\frac{2 \sqrt{6} a_{3}}{3}-\frac{\sqrt{6} a_{4}}{3}$ & $\Xi^{-} \pi^{+} K^{0}$ & $2 a_{3}+2 a_{4}$ & & $\left.-a_{5}-a_{6}\right)$ \\
\hline$\Xi^{-} K^{+} \bar{K}^{0}$ & $-2 a_{3}+2 a_{6}$ & $p \pi^{0} K^{-}$ & $-\sqrt{2} a_{5}-\sqrt{2} a_{6}$ & $n K^{+} K^{-}$ & $4 a_{1}+2 a_{2}+2 a_{3}$ \\
\hline$p K^{-} \bar{K}^{0}$ & $2 a_{6}$ & $p \pi^{-} \bar{K}^{0}$ & $-2 a_{5}$ & $n \eta^{0} \eta^{0}$ & $4 a_{1}+\frac{8 a_{2}}{3}+\frac{8 a_{3}}{3}$ \\
\hline$n \bar{K}^{0} \bar{K}^{0}$ & $4 a_{4}+4 a_{6}$ & $p K^{-} \eta^{0}$ & $\frac{\sqrt{6} a_{5}}{3}+\sqrt{6} a_{6}$ & & $+\frac{8 a_{4}}{3}-\frac{2 a_{5}}{3}$ \\
\hline$\Lambda^{0} \pi^{0} \bar{K}^{0}$ & $-\sqrt{3}\left(\frac{a_{2}}{3}+\frac{2 a_{3}}{3}+a_{4}+\frac{a_{5}}{3}\right)$ & $n \pi^{0} \bar{K}^{0}$ & $\sqrt{2} a_{2}+\sqrt{2} a_{3}+\sqrt{2} a_{5}-\sqrt{2} a_{6}$ & $\Lambda^{0} \pi^{0} K^{0}$ & $-\sqrt{3}\left(\frac{2 a_{2}}{3}+\frac{a_{3}}{3}+\frac{2 a_{5}}{3}\right)$ \\
\hline$\Lambda^{0} \pi^{+} K^{-}$ & $\frac{\sqrt{6} a_{2}}{3}+\frac{2 \sqrt{6} a_{3}}{3}+\frac{\sqrt{6} a_{5}}{3}$ & $\begin{array}{l}n \pi^{+} K^{-} \\
n \bar{K}^{0} \eta^{0} \\
\Lambda^{0} \pi^{0} \pi^{0} \\
\Lambda^{0} \pi^{0} \eta^{0} \\
\Lambda^{0} \pi^{+} \pi^{-} \\
\Lambda^{0} K^{0} \bar{K}^{0} \\
\Lambda^{0} K^{+} K^{-} \\
\Lambda^{0} \eta^{0} \eta^{0}\end{array}$ & $\begin{array}{l}-2 a_{2}-2 a_{3}-2 a_{5} \\
\sqrt{6}\left(\frac{a_{2}}{3}+\frac{a_{3}}{3}+\frac{4 a_{4}}{3}+\frac{a_{5}}{3}+a_{6}\right) \\
\sqrt{6}\left(-2 a_{1}-\frac{2 a_{2}}{3}-\frac{a_{3}}{3}+\frac{a_{5}}{3}\right) \\
\sqrt{2}\left(\frac{2 a_{2}}{3}+\frac{a_{3}}{3}-a_{4}-\frac{a_{5}}{3}-a_{6}\right) \\
\sqrt{6}\left(-2 a_{1}-\frac{2 a_{2}}{3}-\frac{a_{3}}{3}+\frac{a_{5}}{3}\right) \\
\sqrt{6}\left(-2 a_{1}-a_{2}-a_{3}-a_{4}+a_{5}\right) \\
\sqrt{6}\left(-2 a_{1}-\frac{a_{2}}{3}-\frac{2 a_{3}}{3}+\frac{2 a_{5}}{3}\right) \\
\sqrt{6}\left(-2 a_{1}-\frac{2 a_{2}}{3}-a_{3}+\frac{2 a_{4}}{3}\right. \\
\left.+a_{5}+2 a_{6}\right)\end{array}$ & $\Lambda^{0} \pi^{-} K^{+}$ & $\sqrt{6}\left(\frac{2 a_{2}}{3}+\frac{a_{3}}{3}+\frac{2 a_{5}}{3}\right)$ \\
\hline
\end{tabular}

$$
\begin{gathered}
\quad+a_{5}\left(\overline{\mathbf{B}}_{n}\right)_{k}^{l}(M)_{j}^{m}(M)_{m}^{k} H(6)_{i l} T^{i j} \\
\quad+a_{6}\left(\overline{\mathbf{B}}_{n}\right)_{k}^{l}(M)_{j}^{m}(M)_{l}^{k} H(6)_{i m} T^{i j}, \\
B\left(\mathbf{B}_{\mathbf{c}} \rightarrow \mathbf{B}_{n} M M^{\prime}\right) \\
=A\left(\mathbf{B}_{\mathbf{c}} \rightarrow \mathbf{B}_{n} M M^{\prime}\right)\left\{a_{i} \rightarrow b_{i}\right\},
\end{gathered}
$$

respectively, where $T^{i j}=\epsilon^{i j k}\left(\mathbf{B}_{\mathbf{c}}\right)_{k}$. We note that since $\mathrm{CP}$ violating effects in the charmed decays are negligible, the parameters of $a_{i}$ and $b_{i}$ can be taken to be relatively real $[33,34]$, so that there are totally 12 real parameters in the $S U(3)_{f}$ irreducible amplitudes of Eq. (8). Here, we have also assumed that the final state interactions can be ignored. We remark that there are some cases in which the contributions from the s-wave meson-pairs vanish due to the fla- vor structure and Bose statistics, whereas the p-wave ones are dominant, leading to a different set of spin dependent amplitudes, which will not be discussed in this study. For example, the decay of $\Lambda_{c}^{+} \rightarrow \Lambda \pi^{+} \pi^{0}$ with the measured branching ratio around $7.1 \%$ is predicted mainly from the contribution of the p-wave meson-pair. The explicit expansions of $A\left(\Lambda_{c}^{+} \rightarrow \mathbf{B}_{n} M M^{\prime}\right), A\left(\Xi_{c}^{+} \rightarrow \mathbf{B}_{n} M M^{\prime}\right)$ and $A\left(\Xi_{c}^{0} \rightarrow \mathbf{B}_{n} M M^{\prime}\right)$ are presented in Tables 1, 2 and 3, while those of $B\left(\mathbf{B}_{\mathbf{c}} \rightarrow \mathbf{B}_{n} M M^{\prime}\right)$ can be found by replacing $a_{i}$ in $A\left(\mathbf{B}_{\mathbf{c}} \rightarrow \mathbf{B}_{n} M M^{\prime}\right)$ with $b_{i}$, respectively.

The differential decay width with an unpolarized $\mathbf{B}_{\mathbf{c}}$ and up-down asymmetry $\alpha$ in $\mathbf{B}_{\mathbf{c}} \rightarrow \mathbf{B}_{\mathbf{n}} M M^{\prime}$ are given by 
Table 4 The data inputs from Refs. [3,35-38] and reproductions for $\mathcal{B}\left(\Lambda_{c}^{+} \rightarrow \mathbf{B}_{\mathbf{n}} M M\right)$

\begin{tabular}{|c|c|c|c|c|c|}
\hline & Data & Our results & & Data & Our results \\
\hline $10^{2} \mathcal{B}\left(\Lambda_{c}^{+} \rightarrow p K^{-} \pi^{+}\right)$ & $3.4 \pm 0.4$ & $3.4 \pm 0.5$ & $10^{2} \mathcal{B}\left(\Lambda_{c}^{+} \rightarrow p \bar{K}^{0} \eta\right)$ & $1.6 \pm 0.4$ & $0.7 \pm 0.1$ \\
\hline $10^{3} \mathcal{B}\left(\Lambda_{c}^{+} \rightarrow \Lambda^{0} K^{+} \bar{K}^{0}\right)$ & $5.6 \pm 1.1$ & $5.8 \pm 1.0$ & $10^{2} \mathcal{B}\left(\Lambda_{c}^{+} \rightarrow \Sigma^{+} \pi^{0} \pi^{0}\right)$ & $1.3 \pm 0.1$ & $1.3 \pm 0.2$ \\
\hline $10^{2} \mathcal{B}\left(\Lambda_{c}^{+} \rightarrow \Lambda^{0} \pi^{+} \eta\right)$ & $1.8 \pm 0.3$ & $1.7 \pm 0.3$ & $10^{4} \mathcal{B}\left(\Lambda_{c}^{+} \rightarrow p K^{+} \pi^{-}\right)$ & $1.0 \pm 0.1$ & $1.0 \pm 0.1$ \\
\hline $10^{2} \mathcal{B}\left(\Lambda_{c}^{+} \rightarrow \Sigma^{+} \pi^{+} \pi^{-}\right)$ & $4.4 \pm 0.3$ & $4.5 \pm 0.3$ & $10^{2} \mathcal{B}\left(\Xi_{c}^{+} \rightarrow \Xi^{-} \pi^{+} \pi^{+}\right)$ & $4.7 \pm 1.7$ & $5.4 \pm 1.3$ \\
\hline $10^{2} \mathcal{B}\left(\Lambda_{c}^{+} \rightarrow \Sigma^{-} \pi^{+} \pi^{+}\right)$ & $1.9 \pm 0.2$ & $1.9 \pm 0.3$ & $10^{2} \mathcal{B}\left(\Xi_{c}^{0} \rightarrow \Lambda^{0} K^{-} \pi^{+}\right)$ & $1.9 \pm 0.6$ & $2.2 \pm 0.6$ \\
\hline $10^{2} \mathcal{B}\left(\Lambda_{c}^{+} \rightarrow \Sigma^{0} \pi^{+} \pi^{0}\right)$ & $2.2 \pm 0.8$ & $1.0 \pm 0.1$ & $10^{4} \mathcal{B}\left(\Xi_{c}^{0} \rightarrow \Lambda^{0} K^{-} K^{+}\right)$ & $5.2 \pm 1.9$ & $6.2 \pm 1.2$ \\
\hline $10^{3} \mathcal{B}\left(\Lambda_{c}^{+} \rightarrow \Sigma^{+} K^{+} \pi^{-}\right)$ & $2.1 \pm 0.6$ & $2.5 \pm 0.3$ & & & \\
\hline $10^{3} \mathcal{B}\left(\Lambda_{c}^{+} \rightarrow \Xi^{-} K^{+} \pi^{+}\right)$ & $6.2 \pm 0.6$ & $6.1 \pm 0.8$ & & & \\
\hline $10^{3} \mathcal{B}\left(\Lambda_{c}^{+} \rightarrow p \pi^{-} \pi^{+}\right)$ & $4.2 \pm 0.4$ & $4.7 \pm 0.4$ & & & \\
\hline $10^{4} \mathcal{B}\left(\Lambda_{c}^{+} \rightarrow p K^{-} K^{+}\right)$ & $5.2 \pm 1.2$ & $5.0 \pm 1.2$ & & & \\
\hline
\end{tabular}

$$
=\frac{2 \operatorname{Re}\left(S^{*} P\right)}{|S|^{2}+|P|^{2}}
$$

\begin{tabular}{llll}
\hline$a_{i}$ & Result & $b_{i}$ & Result \\
\hline$a_{1}$ & $9.2 \pm 0.7$ & $b_{1}$ & $18.3 \pm 0.9$ \\
$a_{2}$ & $-3.7 \pm 0.5$ & $b_{2}$ & $-9.8 \pm 2.4$ \\
$a_{3}$ & $-7.3 \pm 0.4$ & $b_{3}$ & $4.4 \pm 2.1$ \\
$a_{4}$ & $2.3 \pm 0.4$ & $b_{4}$ & $-5.4 \pm 2.9$ \\
$a_{5}$ & $11.5 \pm 1.3$ & $b_{5}$ & $38.8 \pm 2.2$ \\
$a_{6}$ & $-3.7 \pm 0.2$ & $b_{6}$ & $12.7 \pm 2.3$ \\
\hline
\end{tabular}

$d \Gamma=\frac{1}{(2 \pi)^{3}} \frac{|S|^{2}+|P|^{2}+2 \operatorname{Re}\left(S^{*} P\right) \vec{s}_{\mathbf{B n}} \cdot \vec{p}_{\mathbf{B}_{\mathbf{n}}}}{64 m_{\mathbf{B}_{\mathbf{c}}}^{3}} d m_{12}^{2} d m_{23}^{2}$,

and

$\alpha=\frac{d \Gamma\left(\vec{s}_{\mathbf{B n}} \cdot \vec{p}_{\mathbf{B}_{\mathbf{n}}}=1\right)-d \Gamma\left(\vec{s}_{\mathbf{B n}} \cdot \vec{p}_{\mathbf{B}_{\mathbf{n}}}=-1\right)}{d \Gamma\left(\vec{s}_{\mathbf{B n}} \cdot \vec{p}_{\mathbf{B}_{\mathbf{n}}}=1\right)+d \Gamma\left(\vec{s}_{\mathbf{B n}} \cdot \vec{p}_{\mathbf{B}_{\mathbf{n}}}=-1\right)}$ respectively, where

$S=A, \quad P=\sqrt{\frac{\left(m_{\mathbf{B}_{\mathbf{c}}}-m_{\mathbf{B}_{\mathbf{n}}}\right)^{2}-m_{23}^{2}}{\left(m_{\mathbf{B}_{\mathbf{c}}}+m_{\mathbf{B}_{\mathbf{n}}}\right)^{2}-m_{23}^{2}}} B=\kappa\left(m_{23}^{2}\right) B$,

with $m_{12}=p_{\mathbf{B}_{\mathbf{n}}}+p_{M^{\prime}}, m_{23}=p_{M}+p_{M^{\prime}}$ and $\kappa^{2}\left(m_{23}^{2}\right)=$ $\left(\left(m_{\mathbf{B}_{\mathbf{c}}}-m_{\mathbf{B}_{\mathbf{n}}}\right)^{2}-m_{23}^{2}\right) /\left(\left(m_{\mathbf{B}_{\mathbf{c}}}+m_{\mathbf{B}_{\mathbf{n}}}\right)^{2}-m_{23}^{2}\right)$. In general, $A$ and $B$ in Eq. (8) depend on $m_{12}^{2}$ and $m_{23}^{2}$. However, we can assume them to be constant when the non-resonant contributions are excluded. Consequently, the decay width $\Gamma$ and averaged up-down asymmetry $\langle\alpha\rangle$ can be derived as follows:

$$
\begin{aligned}
\Gamma & =\int_{m_{12}^{2}} \int_{m_{23}^{2}} d \Gamma\left(\vec{s}_{\mathbf{B n}} \cdot \vec{p}_{\mathbf{B}_{\mathbf{n}}}=1\right)+d \Gamma\left(\vec{s}_{\mathbf{B n}} \cdot \vec{p}_{\mathbf{B}_{\mathbf{n}}}=-1\right) \\
& =\int_{m_{12}^{2}} \int_{m_{23}^{2}} \frac{1}{(2 \pi)^{3}} \frac{|S|^{2}+|P|^{2}}{32 m_{\mathbf{B}_{\mathbf{c}}}^{3}} d m_{12}^{2} d m_{23}^{2}
\end{aligned}
$$

Table 6 Numerical results for $\mathcal{B}\left(\Lambda_{c}^{+} \rightarrow \mathbf{B}_{\mathbf{n}} M M^{\prime}\right)$

\begin{tabular}{llllll}
\hline CF mode & $10^{3} \mathcal{B}$ & CS mode & $10^{4} \mathcal{B}$ & DCS mode & $10^{6} \mathcal{B}$ \\
\hline$\Sigma^{+} \pi^{0} \eta^{0}$ & $6.6 \pm 3.4$ & $\Sigma^{+} \pi^{0} K^{0}$ & $9.9 \pm 2.8$ & $\Sigma^{+} K^{0} K^{0}$ & $1.3 \pm 0.5$ \\
$\Sigma^{+} K^{0} \bar{K}^{0}$ & $2.9 \pm 0.7$ & $\Sigma^{+} K^{0} \eta^{0}$ & $0.26 \pm 0.06$ & $\Sigma^{0} K^{0} K^{+}$ & $1.3 \pm 0.5$ \\
$\Sigma^{+} K^{+} K^{-}$ & $2.5 \pm 0.3$ & $\Sigma^{0} \pi^{0} K^{+}$ & $7.8 \pm 2.3$ & $\Sigma^{-} K^{+} K^{+}$ & $1.3 \pm 0.5$ \\
$\Sigma^{+} \eta^{0} \eta^{0}$ & $(3.2 \pm 0.4) \times 10^{-4}$ & $\Sigma^{0} \pi^{+} K^{0}$ & $9.6 \pm 2.7$ & $p \pi^{0} K^{0}$ & $50 \pm 6$ \\
$\Sigma^{0} \pi^{+} \eta^{0}$ & $6.3 \pm 3.2$ & $\Sigma^{0} K^{+} \eta^{0}$ & $0.13 \pm 0.03$ & $p K^{0} \eta^{0}$ & $3.3 \pm 2.7$ \\
$\Sigma^{0} K^{+} \bar{K}^{0}$ & $0.26 \pm 0.09$ & $p \pi^{0} \pi^{0}$ & $24 \pm 2$ & $n \pi^{0} K^{+}$ & $51 \pm 6$ \\
$\Xi^{0} \pi^{0} K^{+}$ & $32 \pm 6$ & $p \pi^{0} \eta^{0}$ & $34 \pm 7$ & $n \pi^{+} K^{0}$ & $99 \pm 11$ \\
$\Xi^{0} \pi^{+} K^{0}$ & $44 \pm 8$ & $p K^{0} \bar{K}^{0}$ & $37 \pm 8$ & $n K^{+} \eta^{0}$ & $3.4 \pm 2.7$ \\
$p \pi^{0} \bar{K}^{0}$ & $23 \pm 4$ & $p \eta^{0} \eta^{0}$ & $2.8 \pm 1.2$ & & \\
$n \pi \bar{K}^{0}$ & $11 \pm 1$ & $n \pi^{+} \eta^{0}$ & $67 \pm 13$ & & \\
& & $n K^{+} \bar{K}^{0}$ & $31 \pm 9$ & & \\
& & $\Lambda^{0} \pi^{0} K^{+}$ & $35 \pm 6$ & & \\
& & $\Lambda^{0} \pi^{+} K^{0}$ & $67 \pm 11$ & & \\
\hline
\end{tabular}


Table 7 Numerical results for $\mathcal{B}\left(\Xi_{c}^{+} \rightarrow \mathbf{B}_{\mathbf{n}} M M^{\prime}\right)$

\begin{tabular}{|c|c|c|c|c|c|}
\hline CF mode & $10^{2} \mathcal{B}$ & CS mode & $10^{3} \mathcal{B}$ & DCS mode & $10^{4} \mathcal{B}$ \\
\hline$\Sigma^{+} \pi^{0} \bar{K}^{0}$ & $2.0 \pm 1.3$ & $\Sigma^{+} \pi^{0} \eta^{0}$ & $5.3 \pm 1.1$ & $\Sigma^{+} \pi^{0} K^{0}$ & $2.6 \pm 0.2$ \\
\hline$\Sigma^{+} \pi^{+} K^{-}$ & $5.4 \pm 0.5$ & $\Sigma^{+} \pi^{+} \pi^{-}$ & $5.9 \pm 1.5$ & $\Sigma^{+} \pi^{-} K^{+}$ & $1.4 \pm 0.3$ \\
\hline$\Sigma^{+} \bar{K}^{0} \eta^{0}$ & $0.30 \pm 0.09$ & $\Sigma^{+} K^{0} \bar{K}^{0}$ & $4.3 \pm 0.9$ & $\Sigma^{+} K^{0} \eta^{0}$ & $0.020 \pm 0.014$ \\
\hline$\Sigma^{0} \pi^{+} \bar{K}^{0}$ & $0.95 \pm 0.21$ & $\Sigma^{+} K^{+} K^{-}$ & $0.58 \pm 0.09$ & $\Sigma^{0} \pi^{0} K^{+}$ & $0.076 \pm 0.059$ \\
\hline$\Xi^{0} \pi^{0} \pi^{+}$ & $1.5 \pm 0.3$ & $\Sigma^{+} \eta^{0} \eta^{0}$ & $0.31 \pm 0.09$ & $\Sigma^{0} \pi^{+} K^{0}$ & $2.5 \pm 0.2$ \\
\hline$\Xi^{0} \pi^{+} \eta^{0}$ & $1.1 \pm 0.3$ & $\Sigma^{0} \pi^{0} \pi^{+}$ & $7.9 \pm 1.4$ & $\Sigma^{0} K^{+} \eta^{0}$ & $0.010 \pm 0.007$ \\
\hline$\Xi^{0} K^{+} \bar{K}^{0}$ & $0.34 \pm 0.06$ & $\Sigma^{0} \pi^{+} \eta^{0}$ & $5.1 \pm 1.1$ & $\Sigma^{-} \pi^{+} K^{+}$ & $1.3 \pm 0.1$ \\
\hline$\Xi^{-} \pi^{+} \pi^{+}$ & $5.7 \pm 1.3$ & $\Sigma^{0} K^{+} \bar{K}^{0}$ & $1.5 \pm 0.5$ & $\Xi^{0} K^{0} K^{+}$ & $0.030 \pm 0.019$ \\
\hline$p \bar{K}^{0} \bar{K}^{0}$ & $3.7 \pm 0.9$ & $\Sigma^{-} \pi^{+} \pi^{+}$ & $16 \pm 3$ & $\Xi^{-} K^{+} K^{+}$ & $0.057 \pm 0.032$ \\
\hline \multirow[t]{11}{*}{$\Lambda^{0} \pi^{+} \bar{K}^{0}$} & $3.8 \pm 0.9$ & $\Xi^{0} \pi^{0} K^{+}$ & $3.6 \pm 0.8$ & $p \pi^{0} \pi^{0}$ & $7.2 \pm 1.8$ \\
\hline & & $\Xi^{0} \pi^{+} K^{0}$ & $8.4 \pm 2.5$ & $p \pi^{0} \eta^{0}$ & $11 \pm 2$ \\
\hline & & $\Xi^{0} K^{+} \eta^{0}$ & $0.42 \pm 0.13$ & $p \pi^{+} \pi^{-}$ & $14 \pm 4$ \\
\hline & & $p \pi^{0} \bar{K}^{0}$ & $19 \pm 3$ & $p K^{0} \bar{K}^{0}$ & $7.7 \pm 1.7$ \\
\hline & & $p \pi^{+} K^{-}$ & $27 \pm 3$ & $p K^{+} K^{-}$ & $1.6 \pm 1.2$ \\
\hline & & $n \pi^{+} \bar{K}^{0}$ & $9.2 \pm 2.0$ & $p \eta^{0} \eta^{0}$ & $0.93 \pm 0.45$ \\
\hline & & $\Lambda^{0} \pi^{+} \eta^{0}$ & $15 \pm 3$ & $n \pi^{+} \eta^{0}$ & $21 \pm 4$ \\
\hline & & $\Lambda^{0} K^{+} \bar{K}^{0}$ & $5.8 \pm 0.8$ & $n K^{+} \bar{K}^{0}$ & $16 \pm 3$ \\
\hline & & & & $\Lambda^{0} \pi^{0} K^{+}$ & $5.0 \pm 1.0$ \\
\hline & & & & $\Lambda^{0} \pi^{+} K^{0}$ & $9.7 \pm 2.0$ \\
\hline & & & & $\Lambda^{0} K^{+} \eta^{0}$ & $0.90 \pm 0.22$ \\
\hline
\end{tabular}

and

$\langle\alpha\rangle \equiv \frac{\int_{m_{12}^{2}} \int_{m_{23}^{2}} d \Gamma\left(\vec{s}_{\mathbf{B n}} \cdot \vec{p}_{\mathbf{B}_{\mathbf{n}}}=1\right)-d \Gamma\left(\vec{s}_{\mathbf{B n}} \cdot \vec{p}_{\mathbf{B}_{\mathbf{n}}}=-1\right)}{\int_{m_{12}^{2}} \int_{m_{23}^{2}} d \Gamma\left(\vec{s}_{\mathbf{B n}} \cdot \vec{p}_{\mathbf{B}_{\mathbf{n}}}=1\right)+d \Gamma\left(\vec{s}_{\mathbf{B n}} \cdot \vec{p}_{\mathbf{B}_{\mathbf{n}}}=-1\right)}$.

\section{Numerical results}

In the numerical analysis, we perform the minimum $\chi^{2}$ fit to obtain the values of $a_{i}$ and $b_{i}$ in in Eq. (8) under $S U(3)_{f}$ for the $\mathbf{B}_{\mathbf{c}} \rightarrow \mathbf{B}_{n} M M^{\prime}$ decays. The $\chi^{2}$ fit is given by

$\chi^{2}=\sum_{i}\left(\frac{\mathcal{B}_{\text {SU(3) }}^{i}-\mathcal{B}_{\text {data }}^{i}}{\sigma_{\text {data }}^{i}}\right)^{2}$,

where $\mathcal{B}_{S U(3)}^{i}$ represents the $i$-th decay branching ratio from the $S U(3)_{f}$ irreducible amplitude, $\mathcal{B}_{\text {data }}^{i}$ stands for the $i$-th experimental data, and $\sigma_{\text {data }}^{i}$ corresponds to the $i$-th experimental error, while $i=1,2, \ldots, 16$ for the 16 measured modes in Table 4. Using $\sin \theta_{c}=0.2248$ [3], one gets that $t_{c}=0.2307$ in Eq. (5).

We now discuss our data input sections in Table 4. First of all, we exclude the resonant contributions from all the data in the table. In particular, we use the non-resonant data of $\Lambda_{c}^{+} \rightarrow p K^{-} \pi^{+}$from the PDG [3]. In addition, we pick up the data for $\Lambda_{c}^{+} \rightarrow p K^{-} K^{+}$and $\Xi_{c}^{0} \rightarrow \Lambda^{0} K^{-} K^{+}$ without the contributions of the resonant process of $\phi \rightarrow$ $K^{+} K^{-}$. For the other $\Lambda_{c}^{+}$decays, their resonant contributions can be taken to be small so that they are insensitive to our fitting results, such as $\mathcal{B}\left(\Lambda_{c}^{+} \rightarrow \Sigma^{+}\left(\rho^{0} \rightarrow\right.\right.$ )$\left.\pi^{+} \pi^{-}\right)<1.7 \%$ [3]. For this reason, we choose the total branching ratios as our data points. The value of $\mathcal{B}\left(\Xi_{c}^{+} \rightarrow\right.$ $\left.\Xi^{-} \pi^{+} \pi^{+}\right)$is extracted from the ratio $\frac{\mathcal{B}\left(\Xi_{c}^{+} \rightarrow \Xi^{0} e^{+} \mu_{e}\right)}{\mathcal{B}\left(\Xi_{c}^{+} \rightarrow \Xi^{-} \pi^{+} \pi^{+}\right)}=$ $2.3_{-0.8}^{+0.7}$ in the PDG [3] with the theoretical prediction of $\mathcal{B}\left(\Xi_{c}^{+} \rightarrow \Xi^{0} e^{+} \mu_{e}\right)=(10.8 \pm 0.9) \%$ by $S U(3)_{f}$ and heavy quark symmetry [27]. For $\Xi_{c}^{0}$ decay processes, the data of $\mathcal{B}\left(\Xi_{c}^{0} \rightarrow \Xi^{-} \pi^{+}\right)=(1.8 \pm 0.5) \%$ measured by BELLE [36] and the ratios of $\frac{\mathcal{B}\left(\Xi_{c}^{0} \rightarrow \Lambda^{0} K^{-} \pi^{+}\right)}{\mathcal{B}\left(\Xi_{c}^{0} \rightarrow \Xi^{-} \pi^{+}\right)}=1.07 \pm 0.14$ and $\frac{\mathcal{B}\left(\Xi_{c}^{0} \rightarrow \Lambda^{0} K^{+} K^{-}\right)}{\mathcal{B}\left(\Xi_{c}^{0} \rightarrow \Xi^{-} \pi^{+}\right)}=0.029 \pm 0.007$ in PDG [3] are used to extract the absolute branching ratios of $\mathcal{B}\left(\Xi_{c}^{0} \rightarrow \Lambda^{0} K^{-} \pi^{+}\right)$ and $\mathcal{B}\left(\Xi_{c}^{0} \rightarrow \Lambda^{0} K^{+} K^{-}\right)$.

There are 12 parameters to be extracted with 16 data inputs as shown in Table 4. In Table 5, we present the fitting values of $a_{i}$ and $b_{i}$. The correlation coefficients of $i$-th and $j$-th irreducible amplitudes are given by 
Table 8 Numerical results for $\mathcal{B}\left(\Xi_{c}^{0} \rightarrow \mathbf{B}_{\mathbf{n}} M M^{\prime}\right)$

\begin{tabular}{|c|c|c|c|c|c|}
\hline CF mode & $10^{2} \mathcal{B}$ & CS mode & $10^{3} \mathcal{B}$ & DCS mode & $10^{5} \mathcal{B}$ \\
\hline$\Sigma^{+} \pi^{0} K^{-}$ & $7.9 \pm 1.4$ & $\Sigma^{+} \pi^{0} \pi^{-}$ & $1.0 \pm 0.2$ & $\Sigma^{+} \pi^{-} K^{0}$ & $4.3 \pm 0.6$ \\
\hline$\Sigma^{+} \pi^{-} \bar{K}^{0}$ & $15 \pm 2$ & $\Sigma^{+} \pi^{-} \eta^{0}$ & $4.5 \pm 0.6$ & $\Sigma^{0} \pi^{0} K^{0}$ & $1.1 \pm 0.4$ \\
\hline$\Sigma^{+} K^{-} \eta^{0}$ & $0.37 \pm 0.07$ & $\Sigma^{+} K^{0} K^{-}$ & $1.8 \pm 0.3$ & $\Sigma^{0} \pi^{-} K^{+}$ & $5.2 \pm 0.6$ \\
\hline$\Sigma^{0} \pi^{0} \bar{K}^{0}$ & $2.4 \pm 0.5$ & $\Sigma^{0} \pi^{0} \pi^{0}$ & $1.8 \pm 0.2$ & $\Sigma^{0} K^{0} \eta^{0}$ & $0.024 \pm 0.016$ \\
\hline$\Sigma^{0} \pi^{+} K^{-}$ & $3.9 \pm 1.0$ & $\Sigma^{0} \pi^{0} \eta^{0}$ & $1.8 \pm 0.2$ & $\Sigma^{-} \pi^{0} K^{+}$ & $5.1 \pm 0.6$ \\
\hline$\Sigma^{0} \bar{K}^{0} \eta^{0}$ & $0.061 \pm 0.015$ & $\Sigma^{0} K^{0} \bar{K}^{0}$ & $0.039 \pm 0.007$ & $\Sigma^{-} \pi^{+} K^{0}$ & $3.1 \pm 0.4$ \\
\hline$\Sigma^{-} \pi^{+} \bar{K}^{0}$ & $0.34 \pm 0.05$ & $\Sigma^{0} K^{+} K^{-}$ & $1.2 \pm 0.2$ & $\Sigma^{-} K^{+} \eta^{0}$ & $0.047 \pm 0.031$ \\
\hline$\Xi^{0} \pi^{0} \pi^{0}$ & $7.2 \pm 1.5$ & $\Sigma^{0} \eta^{0} \eta^{0}$ & $0.39 \pm 0.01$ & $\Xi^{0} K^{0} K^{0}$ & $0.085 \pm 0.031$ \\
\hline$\Xi^{0} \pi^{0} \eta^{0}$ & $1.0 \pm 0.1$ & $\Sigma^{-} \pi^{0} \pi^{+}$ & $1.0 \pm 0.2$ & $\Xi^{-} K^{0} K^{+}$ & $0.040 \pm 0.015$ \\
\hline$\Xi^{0} \pi^{+} \pi^{-}$ & $11 \pm 2$ & $\Sigma^{-} \pi^{+} \eta^{0}$ & $0.44 \pm 0.13$ & $p \pi^{-} \eta^{0}$ & $64 \pm 10$ \\
\hline$\Xi^{0} K^{0} \bar{K}^{0}$ & $0.033 \pm 0.004$ & $\Sigma^{-} K^{+} \bar{K}^{0}$ & $0.24 \pm 0.04$ & $p K^{0} K^{-}$ & $43 \pm 5$ \\
\hline$\Xi^{0} K^{+} K^{-}$ & $0.30 \pm 0.03$ & $\Xi^{0} \pi^{0} K^{0}$ & $0.59 \pm 0.28$ & $n \pi^{0} \pi^{0}$ & $16 \pm 2$ \\
\hline$\Xi^{0} \eta^{0} \eta^{0}$ & $(8.2 \pm 2.5) \times 10^{-4}$ & $\Xi^{0} \pi^{-} K^{+}$ & $1.0 \pm 0.3$ & $n \pi^{0} \eta^{0}$ & $32 \pm 5$ \\
\hline$\Xi^{-} \pi^{0} \pi^{+}$ & $0.37 \pm 0.08$ & $\Xi^{0} K^{0} \eta^{0}$ & $0.013 \pm 0.005$ & $n \pi^{+} \pi^{-}$ & $31 \pm 4$ \\
\hline$\Xi^{-} \pi^{+} \eta^{0}$ & $0.93 \pm 0.11$ & $\Xi^{-} \pi^{0} K^{+}$ & $0.43 \pm 0.11$ & $n K^{0} \bar{K}^{0}$ & $5.0 \pm 0.4$ \\
\hline$\Xi^{-} K^{+} \bar{K}^{0}$ & $0.077 \pm 0.014$ & $\Xi^{-} \pi^{+} K^{0}$ & $0.61 \pm 0.10$ & $n K^{+} K^{-}$ & $22 \pm 4$ \\
\hline$p K^{-} \bar{K}^{0}$ & $1.7 \pm 0.3$ & $\Xi^{-} K^{+} \eta^{0}$ & $(2.3 \pm 0.8) \times 10^{-3}$ & $n \eta^{0} \eta^{0}$ & $0.79 \pm 0.40$ \\
\hline$n \bar{K}^{0} \bar{K}^{0}$ & $0.77 \pm 0.09$ & $p \pi^{0} K^{-}$ & $13 \pm 2$ & $\Lambda^{0} \pi^{0} K^{0}$ & $6.5 \pm 1.9$ \\
\hline$\Lambda^{0} \pi^{+} K^{-}$ & $2.2 \pm 0.6$ & $p \pi^{-} \bar{K}^{0}$ & $22 \pm 4$ & $\Lambda^{0} \pi^{-} K^{+}$ & $13 \pm 4$ \\
\hline \multirow[t]{10}{*}{$\Lambda^{0} \bar{K}^{0} \eta^{0}$} & $0.057 \pm 0.024$ & $p K^{-} \eta^{0}$ & $1.9 \pm 0.4$ & $\Lambda^{0} K^{0} \eta^{0}$ & $0.61 \pm 0.17$ \\
\hline & & $n \pi^{0} \bar{K}^{0}$ & $2.4 \pm 1.2$ & & \\
\hline & & $n \pi^{+} K^{-}$ & $8.9 \pm 2.8$ & & \\
\hline & & $n \bar{K}^{0} \eta^{0}$ & $0.80 \pm 0.29$ & & \\
\hline & & $\Lambda^{0} \pi^{0} \pi^{0}$ & $8.8 \pm 1.1$ & & \\
\hline & & $\Lambda^{0} \pi^{0} \eta^{0}$ & $2.0 \pm 0.3$ & & \\
\hline & & $\Lambda^{0} \pi^{+} \pi^{-}$ & $17 \pm 2$ & & \\
\hline & & $\Lambda^{0} K^{0} \bar{K}^{0}$ & $0.23 \pm 0.03$ & & \\
\hline & & $\Lambda^{0} K^{+} K^{-}$ & $0.62 \pm 0.12$ & & \\
\hline & & $\Lambda^{0} \eta^{0} \eta^{0}$ & $0.18 \pm 0.03$ & & \\
\hline
\end{tabular}

$$
R=\left(\begin{array}{ccccccccccccc}
1 & -0.58 & -0.44 & 0.43 & 0.96 & -0.32 & -0.01 & 0.47 & -0.33 & 0.55 & 0.73 & -0.56 \\
-0.58 & 1 & 0.55 & -0.64 & -0.49 & 0.29 & 0.65 & -0.82 & 0.21 & -0.72 & -0.79 & 0.63 \\
-0.44 & 0.55 & 1 & -0.68 & -0.36 & 0.66 & 0.44 & -0.54 & 0.37 & -0.63 & -0.45 & 0.61 \\
0.43 & -0.64 & -0.68 & 1 & 0.46 & -0.61 & -0.60 & 0.61 & -0.33 & 0.78 & 0.58 & -0.65 \\
0.96 & -0.49 & -0.36 & 0.46 & 1 & -0.27 & 0.00 & 0.41 & -0.23 & 0.55 & 0.74 & -0.52 \\
-0.32 & 0.29 & 0.66 & -0.61 & -0.27 & 1 & 0.28 & -0.34 & 0.50 & -0.46 & -0.23 & 0.54 \\
-0.01 & 0.65 & 0.44 & -0.60 & 0.00 & 0.28 & 1 & -0.79 & 0.38 & -0.76 & -0.44 & 0.69 \\
0.47 & -0.82 & -0.54 & 0.61 & 0.41 & -0.34 & -0.79 & 1 & -0.34 & 0.74 & 0.82 & -0.69 \\
-0.33 & 0.21 & 0.37 & -0.33 & -0.23 & 0.50 & 0.38 & -0.34 & 1 & -0.63 & 0.01 & 0.78 \\
0.55 & -0.72 & -0.63 & 0.78 & 0.55 & -0.46 & -0.76 & 0.74 & -0.63 & 1 & 0.58 & -0.94 \\
0.73 & -0.79 & -0.45 & 0.58 & 0.74 & -0.23 & -0.44 & 0.82 & 0.01 & 0.58 & 1 & -0.48 \\
-0.56 & 0.63 & 0.61 & -0.65 & -0.52 & 0.54 & 0.69 & -0.69 & 0.78 & -0.94 & -0.48 & 1
\end{array}\right) .
$$


Table 9 Numerical results for $\langle\alpha\rangle\left(\Lambda_{c}^{+} \rightarrow \mathbf{B}_{\mathbf{n}} M M^{\prime}\right)$

\begin{tabular}{|c|c|c|c|c|c|}
\hline CF mode & $\langle\alpha\rangle$ & CS mode & $\langle\alpha\rangle$ & DCS mode & $\langle\alpha\rangle$ \\
\hline$\Lambda_{c}^{+} \rightarrow \Sigma^{+} \pi^{0} \pi^{0}$ & $0.85 \pm 0.13$ & $\Lambda_{c}^{+} \rightarrow \Sigma^{+} \pi^{0} K^{0}$ & $0.76 \pm 0.22$ & $\Lambda_{c}^{+} \rightarrow \Sigma^{+} K^{0} K^{0}$ & $-0.43 \pm 0.32$ \\
\hline$\Lambda_{c}^{+} \rightarrow \Sigma^{+} \pi^{0} \eta^{0}$ & $0.81 \pm 0.18$ & $\Lambda_{c}^{+} \rightarrow \Sigma^{+} \pi^{-} K^{+}$ & $0.75 \pm 0.15$ & $\Lambda_{c}^{+} \rightarrow \Sigma^{0} K^{0} K^{+}$ & $-0.43 \pm 0.32$ \\
\hline$\Lambda_{c}^{+} \rightarrow \Sigma^{+} \pi^{+} \pi^{-}$ & $0.16 \pm 0.27$ & $\Lambda_{c}^{+} \rightarrow \Sigma^{+} K^{0} \eta^{0}$ & $-0.05 \pm 0.07$ & $\Lambda_{c}^{+} \rightarrow \Sigma^{-} K^{+} K^{+}$ & $-0.43 \pm 0.31$ \\
\hline$\Lambda_{c}^{+} \rightarrow \Sigma^{+} K^{0} \bar{K}^{0}$ & $0.68 \pm 0.07$ & $\Lambda_{c}^{+} \rightarrow \Sigma^{0} \pi^{0} K^{+}$ & $0.75 \pm 0.10$ & $\Lambda_{c}^{+} \rightarrow p \pi^{0} K^{0}$ & $0.93_{-0.10}^{+0.07}$ \\
\hline$\Lambda_{c}^{+} \rightarrow \Sigma^{+} K^{+} K^{-}$ & $-0.06 \pm 0.11$ & $\Lambda_{c}^{+} \rightarrow \Sigma^{0} \pi^{+} K^{0}$ & $0.75 \pm 0.22$ & $\Lambda_{c}^{+} \rightarrow p \pi^{-} K^{+}$ & $0.93_{-0.10}^{+0.07}$ \\
\hline$\Lambda_{c}^{+} \rightarrow \Sigma^{+} \eta^{0} \eta^{0}$ & $0.03 \pm 0.00$ & $\Lambda_{c}^{+} \rightarrow \Sigma^{0} K^{+} \eta^{0}$ & $-0.05 \pm 0.07$ & $\Lambda_{c}^{+} \rightarrow p K^{0} \eta^{0}$ & $-0.38 \pm 0.45$ \\
\hline$\Lambda_{c}^{+} \rightarrow \Sigma^{0} \pi^{0} \pi^{+}$ & $-0.96_{-0.04}^{+0.07}$ & $\Lambda_{c}^{+} \rightarrow \Sigma^{-} \pi^{+} K^{+}$ & $0.70 \pm 0.70$ & $\Lambda_{c}^{+} \rightarrow n \pi^{0} K^{+}$ & $0.93_{-0.10}^{+0.07}$ \\
\hline$\Lambda_{c}^{+} \rightarrow \Sigma^{0} \pi^{+} \eta^{0}$ & $0.81 \pm 0.18$ & $\Lambda_{c}^{+} \rightarrow p \pi^{0} \pi^{0}$ & $-0.95 \pm 0.05$ & $\Lambda_{c}^{+} \rightarrow n \pi^{+} K^{0}$ & $0.93_{-0.10}^{+0.07}$ \\
\hline$\Lambda_{c}^{+} \rightarrow \Sigma^{0} K^{+} \bar{K}^{0}$ & $0.30 \pm 0.60$ & $\Lambda_{c}^{+} \rightarrow p \pi^{0} \eta^{0}$ & $0.84 \pm 0.09$ & $\Lambda_{c}^{+} \rightarrow n K^{+} \eta^{0}$ & $-0.38 \pm 0.45$ \\
\hline$\Lambda_{c}^{+} \rightarrow \Sigma^{-} \pi^{+} \pi^{+}$ & $-0.96_{-0.04}^{+0.07}$ & $\Lambda_{c}^{+} \rightarrow p \pi^{+} \pi^{-}$ & $-0.95 \pm 0.05$ & & \\
\hline$\Lambda_{c}^{+} \rightarrow \Xi^{0} \pi^{0} K^{+}$ & $0.78 \pm 0.03$ & $\Lambda_{c}^{+} \rightarrow p K^{0} \bar{K}^{0}$ & $0.84 \pm 0.05$ & & \\
\hline$\Lambda_{c}^{+} \rightarrow \Xi^{0} \pi^{+} K^{0}$ & $0.96 \pm 0.00$ & $\Lambda_{c}^{+} \rightarrow p K^{+} K^{-}$ & $-0.91 \pm 0.09$ & & \\
\hline$\Lambda_{c}^{+} \rightarrow \Xi^{-} \pi^{+} K^{+}$ & $-0.78 \pm 0.13$ & $\Lambda_{c}^{+} \rightarrow p \eta^{0} \eta^{0}$ & $0.62 \pm 0.21$ & & \\
\hline$\Lambda_{c}^{+} \rightarrow p \pi^{0} \bar{K}^{0}$ & $0.11 \pm 0.28$ & $\Lambda_{c}^{+} \rightarrow n \pi^{+} \eta^{0}$ & $0.85 \pm 0.09$ & & \\
\hline$\Lambda_{c}^{+} \rightarrow p \pi^{+} K^{-}$ & $0.89 \pm 0.10$ & $\Lambda_{c}^{+} \rightarrow n K^{+} \bar{K}^{0}$ & $0.94 \pm 0.03$ & & \\
\hline$\Lambda_{c}^{+} \rightarrow p \bar{K}^{0} \eta^{0}$ & $-0.38 \pm 0.22$ & $\Lambda_{c}^{+} \rightarrow \Lambda^{0} \pi^{0} K^{+}$ & $0.97 \pm 0.00$ & & \\
\hline$\Lambda_{c}^{+} \rightarrow n \pi^{+} \bar{K}^{0}$ & $-0.91_{-0.09}^{+0.13}$ & $\Lambda_{c}^{+} \rightarrow \Lambda^{0} \pi^{+} K^{0}$ & $0.97 \pm 0.00$ & & \\
\hline$\Lambda_{c}^{+} \rightarrow \Lambda^{0} \pi^{+} \eta^{0}$ & $0.54 \pm 0.15$ & $\Lambda_{c}^{+} \rightarrow \Lambda^{0} K^{+} \eta^{0}$ & $-0.28 \pm 0.28$ & & \\
\hline$\Lambda_{c}^{+} \rightarrow \Lambda^{0} K^{+} \bar{K}^{0}$ & $0.41 \pm 0.08$ & & & & \\
\hline
\end{tabular}

Table 10 Numerical results for $\langle\alpha\rangle\left(\Xi_{c}^{+} \rightarrow \mathbf{B}_{\mathbf{n}} M M^{\prime}\right)$

\begin{tabular}{|c|c|c|c|c|c|}
\hline CF mode & $\langle\alpha\rangle$ & CS mode & $\langle\alpha\rangle$ & DCS mode & $\langle\alpha\rangle$ \\
\hline$\Xi_{c}^{+} \rightarrow \Sigma^{+} \pi^{0} \bar{K}^{0}$ & $0.67 \pm 0.22$ & $\Xi_{c}^{+} \rightarrow \Sigma^{+} \pi^{0} \pi^{0}$ & $-0.95_{-0.05}^{+0.65}$ & $\Xi_{c}^{+} \rightarrow \Sigma^{+} \pi^{0} K^{0}$ & $-0.26 \pm 0.15$ \\
\hline$\Xi_{c}^{+} \rightarrow \Sigma^{+} \pi^{+} K^{-}$ & $0.86_{-0.15}^{+0.14}$ & $\Xi_{c}^{+} \rightarrow \Sigma^{+} \pi^{0} \eta^{0}$ & $0.33 \pm 0.19$ & $\Xi_{c}^{+} \rightarrow \Sigma^{+} \pi^{-} K^{+}$ & $0.80 \pm 0.13$ \\
\hline$\Xi_{c}^{+} \rightarrow \Sigma^{+} \bar{K}^{0} \eta^{0}$ & $0.21 \pm 0.10$ & $\Xi_{c}^{+} \rightarrow \Sigma^{+} \pi^{+} \pi^{-}$ & $-0.96 \pm 0.04$ & $\Xi_{c}^{+} \rightarrow \Sigma^{+} K^{0} \eta^{0}$ & $0.61 \pm 0.33$ \\
\hline$\Xi_{c}^{+} \rightarrow \Sigma^{0} \pi^{+} \bar{K}^{0}$ & $-0.81_{-0.19}^{+0.36}$ & $\Xi_{c}^{+} \rightarrow \Sigma^{+} K^{0} \bar{K}^{0}$ & $0.71 \pm 0.06$ & $\Xi_{c}^{+} \rightarrow \Sigma^{0} \pi^{0} K^{+}$ & $-0.05 \pm 0.21$ \\
\hline$\Xi_{c}^{+} \rightarrow \Xi^{0} \pi^{0} \pi^{+}$ & $-0.81_{-0.19}^{+0.36}$ & $\Xi_{c}^{+} \rightarrow \Sigma^{+} K^{+} K^{-}$ & $-0.87 \pm 0.11$ & $\Xi_{c}^{+} \rightarrow \Sigma^{0} \pi^{+} K^{0}$ & $-0.26 \pm 0.15$ \\
\hline$\Xi_{c}^{+} \rightarrow \Xi^{0} \pi^{+} \eta^{0}$ & $0.96 \pm 0.04$ & $\Xi_{c}^{+} \rightarrow \Sigma^{+} \eta^{0} \eta^{0}$ & $0.63 \pm 0.09$ & $\Xi_{c}^{+} \rightarrow \Sigma^{0} K^{+} \eta^{0}$ & $0.60 \pm 0.33$ \\
\hline$\Xi_{c}^{+} \rightarrow \Xi^{0} K^{+} \bar{K}^{0}$ & $0.54 \pm 0.17$ & $\Xi_{c}^{+} \rightarrow \Sigma^{0} \pi^{0} \pi^{+}$ & $-0.97 \pm 0.03$ & $\Xi_{c}^{+} \rightarrow \Sigma^{-} \pi^{+} K^{+}$ & $-0.94_{-0.06}^{+0.07}$ \\
\hline$\Xi_{c}^{+} \rightarrow \Xi^{-} \pi^{+} \pi^{+}$ & $-0.81_{-0.19}^{+0.37}$ & $\Xi_{c}^{+} \rightarrow \Sigma^{0} \pi^{+} \eta^{0}$ & $0.34 \pm 0.19$ & $\Xi_{c}^{+} \rightarrow \Xi^{0} K^{0} K^{+}$ & $-0.84_{-0.16}^{+0.18}$ \\
\hline$\Xi_{c}^{+} \rightarrow p \bar{K}^{0} \bar{K}^{0}$ & $-0.87_{-0.13}^{+0.31}$ & $\Xi_{c}^{+} \rightarrow \Sigma^{0} K^{+} \bar{K}^{0}$ & $0.96 \pm 0.01$ & $\Xi_{c}^{+} \rightarrow \Xi^{-} K^{+} K^{+}$ & $-0.83_{-0.17}^{+0.18}$ \\
\hline \multirow[t]{11}{*}{$\Xi_{c}^{+} \rightarrow \Lambda^{0} \pi^{+} \bar{K}^{0}$} & $-0.86_{-0.14}^{+0.33}$ & $\Xi_{c}^{+} \rightarrow \Sigma^{-} \pi^{+} \pi^{+}$ & $-0.96 \pm 0.03$ & $\Xi_{c}^{+} \rightarrow p \pi^{0} \pi^{0}$ & $-0.22 \pm 0.38$ \\
\hline & & $\Xi_{c}^{+} \rightarrow \Xi^{0} \pi^{0} K^{+}$ & $0.95 \pm 0.03$ & $\Xi_{c}^{+} \rightarrow p \pi^{0} \eta^{0}$ & $0.97 \pm 0.00$ \\
\hline & & $\Xi_{c}^{+} \rightarrow \Xi^{0} \pi^{+} K^{0}$ & $0.96 \pm 0.01$ & $\Xi_{c}^{+} \rightarrow p \pi^{+} \pi^{-}$ & $-0.22 \pm 0.38$ \\
\hline & & $\Xi_{c}^{+} \rightarrow \Xi^{0} K^{+} \eta^{0}$ & $0.85 \pm 0.08$ & $\Xi_{c}^{+} \rightarrow p K^{0} \bar{K}^{0}$ & $0.96 \pm 0.02$ \\
\hline & & $\Xi_{c}^{+} \rightarrow \Xi^{-} \pi^{+} K^{+}$ & $0.70_{-0.70}^{+0.30}$ & $\Xi_{c}^{+} \rightarrow p K^{+} K^{-}$ & $0.14 \pm 0.24$ \\
\hline & & $\Xi_{c}^{+} \rightarrow p \pi^{0} \bar{K}^{0}$ & $0.30 \pm 0.17$ & $\Xi_{c}^{+} \rightarrow p \eta^{0} \eta^{0}$ & $0.93_{-0.12}^{+0.07}$ \\
\hline & & $\Xi_{c}^{+} \rightarrow p \pi^{+} K^{-}$ & $0.94_{-0.07}^{+0.06}$ & $\Xi_{c}^{+} \rightarrow n \pi^{+} \eta^{0}$ & $0.97 \pm 0.00$ \\
\hline & & $\Xi_{c}^{+} \rightarrow p \bar{K}^{0} \eta^{0}$ & $0.49 \pm 0.28$ & $\Xi_{c}^{+} \rightarrow n K^{+} \bar{K}^{0}$ & $0.83 \pm 0.06$ \\
\hline & & $\Xi_{c}^{+} \rightarrow n \pi^{+} \bar{K}^{0}$ & $-0.97 \pm 0.02$ & $\Xi_{c}^{+} \rightarrow \Lambda^{0} \pi^{0} K^{+}$ & $0.80 \pm 0.12$ \\
\hline & & $\Xi_{c}^{+} \rightarrow \Lambda^{0} \pi^{+} \eta^{0}$ & $0.96 \pm 0.02$ & $\Xi_{c}^{+} \rightarrow \Lambda^{0} \pi^{+} K^{0}$ & $0.80 \pm 0.12$ \\
\hline & & $\Xi_{c}^{+} \rightarrow \Lambda^{0} K^{+} \bar{K}^{0}$ & $0.91 \pm 0.06$ & $\Xi_{c}^{+} \rightarrow \Lambda^{0} K^{+} \eta^{0}$ & $-0.03 \pm 0.35$ \\
\hline
\end{tabular}


Table 11 Numerical results for $\langle\alpha\rangle\left(\Xi_{c}^{0} \rightarrow \mathbf{B}_{\mathbf{n}} M M^{\prime}\right)$

\begin{tabular}{|c|c|c|c|c|c|}
\hline CF mode & $\langle\alpha\rangle$ & CS mode & $\langle\alpha\rangle$ & DCS mode & $\langle\alpha\rangle$ \\
\hline$\Xi_{c}^{0} \rightarrow \Sigma^{+} \pi^{0} K^{-}$ & $0.94 \pm 0.02$ & $\Xi_{c}^{0} \rightarrow \Sigma^{+} \pi^{0} \pi^{-}$ & $-0.97 \pm 0.03$ & $\Xi_{c}^{0} \rightarrow \Sigma^{+} \pi^{-} K^{0}$ & $-0.94_{-0.06}^{+0.07}$ \\
\hline$\Xi_{c}^{0} \rightarrow \Sigma^{+} \pi^{-} \bar{K}^{0}$ & $0.89 \pm 0.05$ & $\Xi_{c}^{0} \rightarrow \Sigma^{+} \pi^{-} \eta^{0}$ & $0.73 \pm 0.10$ & $\Xi_{c}^{0} \rightarrow \Sigma^{0} \pi^{0} K^{0}$ & $-0.05 \pm 0.21$ \\
\hline$\Xi_{c}^{0} \rightarrow \Sigma^{+} K^{-} \eta^{0}$ & $0.79 \pm 0.03$ & $\Xi_{c}^{0} \rightarrow \Sigma^{+} K^{0} K^{-}$ & $0.82 \pm 0.03$ & $\Xi_{c}^{0} \rightarrow \Sigma^{0} \pi^{-} K^{+}$ & $-0.26 \pm 0.15$ \\
\hline$\Xi_{c}^{0} \rightarrow \Sigma^{0} \pi^{0} \bar{K}^{0}$ & $0.44 \pm 0.17$ & $\Xi_{c}^{0} \rightarrow \Sigma^{0} \pi^{0} \pi^{0}$ & $-0.96 \pm 0.02$ & $\Xi_{c}^{0} \rightarrow \Sigma^{0} K^{0} \eta^{0}$ & $0.60 \pm 0.33$ \\
\hline$\Xi_{c}^{0} \rightarrow \Sigma^{0} \pi^{+} K^{-}$ & $0.95 \pm 0.03$ & $\Xi_{c}^{0} \rightarrow \Sigma^{0} \pi^{0} \eta^{0}$ & $0.89 \pm 0.06$ & $\Xi_{c}^{0} \rightarrow \Sigma^{-} \pi^{0} K^{+}$ & $-0.26 \pm 0.15$ \\
\hline$\Xi_{c}^{0} \rightarrow \Sigma^{0} \bar{K}^{0} \eta^{0}$ & $0.96 \pm 0.01$ & $\Xi_{c}^{0} \rightarrow \Sigma^{0} \pi^{+} \pi^{-}$ & $-0.95_{-0.05}^{+0.64}$ & $\Xi_{c}^{0} \rightarrow \Sigma^{-} \pi^{+} K^{0}$ & $0.80 \pm 0.13$ \\
\hline$\Xi_{c}^{0} \rightarrow \Sigma^{-} \pi^{+} \bar{K}^{0}$ & $-0.96_{-0.04}^{+0.06}$ & $\Xi_{c}^{0} \rightarrow \Sigma^{0} K^{0} \bar{K}^{0}$ & $0.95 \pm 0.05$ & $\Xi_{c}^{0} \rightarrow \Sigma^{-} K^{+} \eta^{0}$ & $0.60 \pm 0.33$ \\
\hline$\Xi_{c}^{0} \rightarrow \Xi^{0} \pi^{0} \pi^{0}$ & $0.86 \pm 0.05$ & $\Xi_{c}^{0} \rightarrow \Sigma^{0} K^{+} K^{-}$ & $0.54 \pm 0.02$ & $\Xi_{c}^{0} \rightarrow \Xi^{0} K^{0} K^{0}$ & $-0.84_{-0.16}^{+0.18}$ \\
\hline$\Xi_{c}^{0} \rightarrow \Xi^{0} \pi^{0} \eta^{0}$ & $0.42 \pm 0.18$ & $\Xi_{c}^{0} \rightarrow \Sigma^{0} \eta^{0} \eta^{0}$ & $0.63 \pm 0.09$ & $\Xi_{c}^{0} \rightarrow \Xi^{-} K^{0} K^{+}$ & $-0.83_{-0.17}^{+0.18}$ \\
\hline$\Xi_{c}^{0} \rightarrow \Xi^{0} \pi^{+} \pi^{-}$ & $0.97 \pm 0.01$ & $\Xi_{c}^{0} \rightarrow \Sigma^{-} \pi^{0} \pi^{+}$ & $-0.97 \pm 0.03$ & $\Xi_{c}^{0} \rightarrow p \pi^{-} \eta^{0}$ & $0.97 \pm 0.00$ \\
\hline$\Xi_{c}^{0} \rightarrow \Xi^{0} K^{0} \bar{K}^{0}$ & $0.32 \pm 0.52$ & $\Xi_{c}^{0} \rightarrow \Sigma^{-} \pi^{+} \eta^{0}$ & $0.78 \pm 0.10$ & $\Xi_{c}^{0} \rightarrow p K^{0} K^{-}$ & $0.83 \pm 0.06$ \\
\hline$\Xi_{c}^{0} \rightarrow \Xi^{0} K^{+} K^{-}$ & $-0.07 \pm 0.13$ & $\Xi_{c}^{0} \rightarrow \Sigma^{-} K^{+} \bar{K}^{0}$ & $0.07 \pm 0.16$ & $\Xi_{c}^{0} \rightarrow n \pi^{0} \pi^{0}$ & $-0.22 \pm 0.38$ \\
\hline$\Xi_{c}^{0} \rightarrow \Xi^{0} \eta^{0} \eta^{0}$ & $-0.18 \pm 0.83$ & $\Xi_{c}^{0} \rightarrow \Xi^{0} \pi^{0} K^{0}$ & $0.37 \pm 0.32$ & $\Xi_{c}^{0} \rightarrow n \pi^{0} \eta^{0}$ & $0.97 \pm 0.00$ \\
\hline$\Xi_{c}^{0} \rightarrow \Xi^{-} \pi^{0} \pi^{+}$ & $-0.81_{-0.19}^{+0.37}$ & $\Xi_{c}^{0} \rightarrow \Xi^{0} \pi^{-} K^{+}$ & $0.16 \pm 0.33$ & $\Xi_{c}^{0} \rightarrow n \pi^{+} \pi^{-}$ & $-0.22 \pm 0.38$ \\
\hline$\Xi_{c}^{0} \rightarrow \Xi^{-} \pi^{+} \eta^{0}$ & $-0.09 \pm 0.11$ & $\Xi_{c}^{0} \rightarrow \Xi^{0} K^{0} \eta^{0}$ & $0.16 \pm 0.15$ & $\Xi_{c}^{0} \rightarrow n K^{0} \bar{K}^{0}$ & $0.14 \pm 0.24$ \\
\hline$\Xi_{c}^{0} \rightarrow \Xi^{-} K^{+} \bar{K}^{0}$ & $0.47 \pm 0.12$ & $\Xi_{c}^{0} \rightarrow \Xi^{-} \pi^{0} K^{+}$ & $0.18 \pm 0.15$ & $\Xi_{c}^{0} \rightarrow n K^{+} K^{-}$ & $0.96 \pm 0.02$ \\
\hline$\Xi_{c}^{0} \rightarrow p K^{-} \bar{K}^{0}$ & $-0.96 \pm 0.03$ & $\Xi_{c}^{0} \rightarrow \Xi^{-} \pi^{+} K^{0}$ & $0.08 \pm 0.19$ & $\Xi_{c}^{0} \rightarrow n \eta^{0} \eta^{0}$ & $0.93_{-0.07}^{+0.12}$ \\
\hline$\Xi_{c}^{0} \rightarrow n \bar{K}^{0} \bar{K}^{0}$ & $-0.93_{-0.07}^{+0.11}$ & $\Xi_{c}^{0} \rightarrow \Xi^{-} K^{+} \eta^{0}$ & $-0.83_{-0.17}^{+0.21}$ & $\Xi_{c}^{0} \rightarrow \Lambda^{0} \pi^{0} K^{0}$ & $0.80 \pm 0.12$ \\
\hline$\Xi_{c}^{0} \rightarrow \Lambda^{0} \pi^{0} \bar{K}^{0}$ & $0.01 \pm 0.62$ & $\Xi_{c}^{0} \rightarrow p \pi^{0} K^{-}$ & $0.75 \pm 0.07$ & $\Xi_{c}^{0} \rightarrow \Lambda^{0} \pi^{-} K^{+}$ & $0.80 \pm 0.12$ \\
\hline$\Xi_{c}^{0} \rightarrow \Lambda^{0} \pi^{+} K^{-}$ & $-0.91 \pm 0.08$ & $\Xi_{c}^{0} \rightarrow p \pi^{-} \bar{K}^{0}$ & $0.97 \pm 0.00$ & $\Xi_{c}^{0} \rightarrow \Lambda^{0} K^{0} \eta^{0}$ & $-0.03 \pm 0.35$ \\
\hline \multirow[t]{10}{*}{$\Xi_{c}^{0} \rightarrow \Lambda^{0} \bar{K}^{0} \eta^{0}$} & $-0.74 \pm 0.22$ & $\Xi_{c}^{0} \rightarrow p K^{-} \eta^{0}$ & $0.04 \pm 0.14$ & & \\
\hline & & $\Xi_{c}^{0} \rightarrow n \pi^{0} \bar{K}^{0}$ & $0.88_{-0.14}^{+0.12}$ & & \\
\hline & & $\Xi_{c}^{0} \rightarrow n \pi^{+} K^{-}$ & $0.10 \pm 0.20$ & & \\
\hline & & $\Xi_{c}^{0} \rightarrow n \bar{K}^{0} \eta^{0}$ & $-0.23 \pm 0.33$ & & \\
\hline & & $\Xi_{c}^{0} \rightarrow \Lambda^{0} \pi^{0} \pi^{0}$ & $0.82 \pm 0.04$ & & \\
\hline & & $\Xi_{c}^{0} \rightarrow \Lambda^{0} \pi^{0} \eta^{0}$ & $0.96 \pm 0.02$ & & \\
\hline & & $\Xi_{c}^{0} \rightarrow \Lambda^{0} \pi^{+} \pi^{-}$ & $0.82 \pm 0.04$ & & \\
\hline & & $\Xi_{c}^{0} \rightarrow \Lambda^{0} K^{0} \bar{K}^{0}$ & $0.92_{-0.09}^{+0.08}$ & & \\
\hline & & $\Lambda^{0} K^{+} K^{-}$ & $0.71 \pm 0.15$ & & \\
\hline & & $\Xi_{c}^{0} \rightarrow \Lambda^{0} \eta^{0} \eta^{0}$ & $-0.90 \pm 0.10$ & & \\
\hline
\end{tabular}

In our fit, we find that $\chi^{2} /$ d.o. $f=9.6 / 4=2.4$ with $d . o . f$ representing degree of freedom. As seen from Table 4 , the decay branching ratios are reproduced, which agree well with the data in Refs. [3,35-38] accordingly.

In Tables 6, 7 and 8, we show our numerical results for the decay branching ratios in $\Lambda_{c}^{+} \rightarrow \mathbf{B}_{\mathbf{n}} M M^{\prime}, \Xi_{c}^{+} \rightarrow \mathbf{B}_{\mathbf{n}} M M^{\prime}$ and $\Xi_{c}^{0} \rightarrow \mathbf{B}_{\mathbf{n}} M M^{\prime}$, respectively.

Since $\left(a_{i}, b_{i}\right)$ and $\left(-a_{i}, b_{i}\right)$ give the same results in our $\chi^{2}$ fitting with $S U(3)_{f}$, both $\pm\langle\alpha\rangle$ are solutions which should be determined by experiments or dynamical models. We present the predictions for the up-down asymmetries of $\langle\alpha\rangle\left(\Lambda_{c}^{+}, \Xi_{c}^{+}, \Xi_{c}^{0} \rightarrow \mathbf{B}_{\mathbf{n}} M M^{\prime}\right)$ in Tables 9,10 and 11 , respectively, by choosing $\langle\alpha\rangle\left(\Lambda_{c}^{+} \rightarrow \Xi^{-} K^{+} \pi^{+}\right)$to be negative. One may also re-parametrize the real $S U(3)$ irreducible amplitudes with $a_{i}$ and $b_{i}$ in Eq. (8) into complex ones with $\tilde{a}_{i}=a_{i}+i \kappa\left(m_{23}^{2}\right) b_{i}$ and assume $\kappa\left(m_{23}^{2}\right)$ being the same for all modes in the $S U(3)_{f}$ limit. In this case, one more parameter can be reduced by considering the following transformations in $a_{i}$ and $b_{i}$ without changing the branching ratios, given by

$$
\begin{aligned}
a_{i}^{\prime} & =\cos (\theta) a_{i}+\kappa \sin (\theta) b_{i}, \\
\kappa b_{i}^{\prime} & =-\sin (\theta) a_{i}+\kappa \cos (\theta) b_{i},
\end{aligned}
$$

which are equivalent to multiply an arbitrary overall phase $e^{i \theta}$ in the complex number parametrization, but lose all information about the up-down asymmetries [25]. Note that the fitting results of the branching ratios are slightly different from those in Ref. [25] due to the kinematic $\kappa\left(m_{23}^{2}\right)$ corrections. The situation also occurs in the semi-leptonic charmed 
Table 12 Decay branching ratios and averaged up-down asymmetries for CF and DCS mixed processes involving $K_{S}^{0}$ and $K_{L}^{0}$

\begin{tabular}{|c|c|c|c|c|c|}
\hline Channel & $\mathcal{B}$ & $\langle\alpha\rangle$ & Channel & $\mathcal{B}$ & $\langle\alpha\rangle$ \\
\hline$\Lambda_{c}^{+} \rightarrow \Sigma^{0} K^{+} K_{S}^{0}$ & $(1.44 \pm 0.52) \times 10^{-4}$ & $0.42 \pm 0.55$ & $\Xi_{c}^{0} \rightarrow \Sigma^{+} \pi^{-} K_{S}^{0}$ & $(7.43 \pm 1.01) \times 10^{-2}$ & $0.90 \pm 0.05$ \\
\hline$\Lambda_{c}^{+} \rightarrow \Sigma^{0} K^{+} K_{L}^{0}$ & $(1.47 \pm 0.51) \times 10^{-4}$ & $0.16 \pm 0.61$ & $\Xi_{c}^{0} \rightarrow \Sigma^{+} \pi^{-} K_{L}^{0}$ & $(7.48 \pm 1.01) \times 10^{-2}$ & $0.87 \pm 0.06$ \\
\hline$\Lambda_{c}^{+} \rightarrow p \pi^{0} K_{S}^{0}$ & $(1.26 \pm 0.21) \times 10^{-2}$ & $0.16 \pm 0.28$ & $\Xi_{c}^{0} \rightarrow \Sigma^{0} \pi^{0} K_{S}^{0}$ & $(1.19 \pm 0.25) \times 10^{-2}$ & $0.44 \pm 0.17$ \\
\hline$\Lambda_{c}^{+} \rightarrow p \pi^{0} K_{L}^{0}$ & $(1.09 \pm 0.19) \times 10^{-2}$ & $0.06 \pm 0.29$ & $\Xi_{c}^{0} \rightarrow \Sigma^{0} \pi^{0} K_{L}^{0}$ & $(1.29 \pm 0.25) \times 10^{-2}$ & $0.43 \pm 0.17$ \\
\hline$\Lambda_{c}^{+} \rightarrow p \eta^{0} K_{S}^{0}$ & $(3.40 \pm 0.54) \times 10^{-3}$ & $-0.35 \pm 0.21$ & $\Xi_{c}^{0} \rightarrow \Sigma^{-} \pi^{+} K_{S}^{0}$ & $(1.84 \pm 0.29) \times 10^{-3}$ & $-0.97 \pm 0.02$ \\
\hline$\Lambda_{c}^{+} \rightarrow p \eta^{0} K_{L}^{0}$ & $(3.52 \pm 0.56) \times 10^{-3}$ & $-0.42 \pm 0.23$ & $\Xi_{c}^{0} \rightarrow \Sigma^{-} \pi^{+} K_{L}^{0}$ & $(1.69 \pm 0.23) \times 10^{-3}$ & $-0.92_{-0.08}^{+0.14}$ \\
\hline$\Lambda_{c}^{+} \rightarrow n \pi^{+} K_{S}^{0}$ & $(5.80 \pm 0.72) \times 10^{-3}$ & $-0.96_{-0.04}^{+0.06}$ & $\Xi_{c}^{0} \rightarrow \Xi^{-} K^{+} K_{S}^{0}$ & $(4.20 \pm 0.73) \times 10^{-4}$ & $0.45 \pm 0.12$ \\
\hline$\Lambda_{c}^{+} \rightarrow n \pi^{+} K_{L}^{0}$ & $(5.88 \pm 0.77) \times 10^{-3}$ & $-0.83_{-0.17}^{+0.20}$ & $\Xi_{c}^{0} \rightarrow \Xi^{-} K^{+} K_{L}^{0}$ & $(3.93 \pm 0.73) \times 10^{-4}$ & $0.51 \pm 0.12$ \\
\hline$\Lambda_{c}^{+} \rightarrow \Sigma^{+} K_{S}^{0} K_{S}^{0}$ & $(1.77 \pm 0.42) \times 10^{-3}$ & $0.69 \pm 0.07$ & $\Xi_{c}^{0} \rightarrow p K^{-} K_{S}^{0}$ & $(8.18 \pm 1.13) \times 10^{-3}$ & $-0.89_{-0.11}^{+0.12}$ \\
\hline$\Lambda_{c}^{+} \rightarrow \Sigma^{+} K_{S}^{0} K_{L}^{0}$ & $(7.56 \pm 2.94) \times 10^{-7}$ & $-0.44 \pm 0.32$ & $\Xi_{c}^{0} \rightarrow p K^{-} K_{L}^{0}$ & $(9.42 \pm 2.00) \times 10^{-3}$ & $-0.95 \pm 0.05$ \\
\hline$\Lambda_{c}^{+} \rightarrow \Sigma^{+} K_{L}^{0} K_{L}^{0}$ & $(1.68 \pm 0.41) \times 10^{-3}$ & $0.71 \pm 0.07$ & $\Xi_{c}^{0} \rightarrow \Lambda^{0} \eta^{0} K_{S}^{0}$ & $(2.69 \pm 1.16) \times 10^{-4}$ & $-0.61 \pm 0.28$ \\
\hline$\Xi_{c}^{+} \rightarrow \Sigma^{+} \pi^{0} K_{S}^{0}$ & $(1.10 \pm 0.66) \times 10^{-2}$ & $0.79 \pm 0.20$ & $\Xi_{c}^{0} \rightarrow \Lambda^{0} \eta^{0} K_{L}^{0}$ & $(3.23 \pm 1.27) \times 10^{-4}$ & $-0.84 \pm 0.16$ \\
\hline$\Xi_{c}^{+} \rightarrow \Sigma^{+} \pi^{0} K_{L}^{0}$ & $(1.00 \pm 0.70) \times 10^{-2}$ & $0.52 \pm 0.23$ & $\Xi_{c}^{0} \rightarrow \Xi^{0} K_{S}^{0} K_{S}^{0}$ & $(1.85 \pm 0.21) \times 10^{-4}$ & $0.37 \pm 0.49$ \\
\hline$\Xi_{c}^{+} \rightarrow \Sigma^{+} \eta^{0} K_{S}^{0}$ & $(1.60 \pm 0.45) \times 10^{-3}$ & $0.22 \pm 0.10$ & $\Xi_{c}^{0} \rightarrow \Xi^{0} K_{S}^{0} K_{L}^{0}$ & $(4.70 \pm 1.71) \times 10^{-7}$ & $-0.85_{-0.15}^{+0.17}$ \\
\hline$\Xi_{c}^{+} \rightarrow \Sigma^{+} \eta^{0} K_{L}^{0}$ & $(1.45 \pm 0.47) \times 10^{-3}$ & $0.20 \pm 0.11$ & $\Xi_{c}^{0} \rightarrow \Xi^{0} K_{L}^{0} K_{L}^{0}$ & $(1.95 \pm 0.19) \times 10^{-4}$ & $0.25 \pm 0.53$ \\
\hline$\Xi_{c}^{+} \rightarrow \Sigma^{0} \pi^{+} K_{S}^{0}$ & $(3.60 \pm 0.90) \times 10^{-3}$ & $-0.87_{-0.13}^{+0.33}$ & $\Xi_{c}^{0} \rightarrow n K_{S}^{0} K_{S}^{0}$ & $(1.66 \pm 0.23) \times 10^{-3}$ & $-0.96 \pm 0.04$ \\
\hline$\Xi_{c}^{+} \rightarrow \Sigma^{0} \pi^{+} K_{L}^{0}$ & $(6.20 \pm 0.13) \times 10^{-3}$ & $-0.76_{-0.24}^{+0.37}$ & $\Xi_{c}^{0} \rightarrow n K_{S}^{0} K_{L}^{0}$ & $(3.97 \pm 0.49) \times 10^{-3}$ & $-0.93_{-0.07}^{+0.12}$ \\
\hline$\Xi_{c}^{+} \rightarrow \Xi^{0} K^{+} K_{S}^{0}$ & $(1.85 \pm 0.34) \times 10^{-3}$ & $0.52 \pm 0.17$ & $\Xi_{c}^{0} \rightarrow n K_{L}^{0} K_{L}^{0}$ & $(2.35 \pm 0.27) \times 10^{-3}$ & $-0.88_{-0.14}^{+0.16}$ \\
\hline$\Xi_{c}^{+} \rightarrow \Xi^{0} K^{+} K_{L}^{0}$ & $(1.75 \pm 0.34) \times 10^{-3}$ & $0.57 \pm 0.17$ & & & \\
\hline$\Xi_{c}^{+} \rightarrow \Lambda^{0} \pi^{+} K_{S}^{0}$ & $(1.94 \pm 0.43) \times 10^{-2}$ & $-0.73_{-0.23}^{+0.47}$ & & & \\
\hline$\Xi_{c}^{+} \rightarrow \Lambda^{0} \pi^{+} K_{L}^{0}$ & $(1.99 \pm 0.49) \times 10^{-2}$ & $-0.94_{0.06}^{0.17}$ & & & \\
\hline$\Xi_{c}^{+} \rightarrow p K_{S}^{0} K_{S}^{0}$ & $(1.06 \pm 0.23) \times 10^{-2}$ & $-0.65_{-0.35}^{+0.50}$ & & & \\
\hline$\Xi_{c}^{+} \rightarrow p K_{S}^{0} K_{L}^{0}$ & $(1.92 \pm 0.44) \times 10^{-2}$ & $-0.88_{-0.12}^{+0.31}$ & & & \\
\hline$\Xi_{c}^{+} \rightarrow p K_{L}^{0} K_{L}^{0}$ & $(9.36 \pm 3.07) \times 10^{-3}$ & $-0.97 \pm 0.01$ & & & \\
\hline
\end{tabular}

baryon decays, indicating that $S U(3)_{f}$ is highly brokien in kinematics [27].

We can also calculate the up-down asymmetries for the decays with the final states involving the physical $K_{S}^{0}$ and $K_{L}^{0}$ particles, where $K_{S}^{0}=\frac{1}{\sqrt{2}}\left(K^{0}+\bar{K}^{0}\right)$ and $K_{L}^{0}=$ $\frac{1}{\sqrt{2}}\left(K^{0}-\bar{K}^{0}\right)$ with ignoring $\mathrm{CP}$ violation. The numerical values for the decay branching ratios and up-down asymmetries are presented in Table 12. It is interesting to note that $\langle\alpha\rangle\left(\Lambda_{c}^{+} \rightarrow \Sigma^{+} K_{S}^{0} K_{L}^{0}\right)=-0.44 \pm 0.32$ and $\langle\alpha\rangle\left(\Xi_{c}^{0} \rightarrow \Xi^{0} K_{S}^{0} K_{L}^{0}\right)=-0.85_{-0.15}^{+0.17}$ are the same as $\langle\alpha\rangle\left(\Lambda_{c}^{+} \rightarrow \Sigma^{+} K^{0} K^{0}\right)$ and $\langle\alpha\rangle\left(\Xi_{c}^{0} \rightarrow \Xi^{0} K^{0} K^{0}\right)$, in which the former two modes are dominated by the CF processes, whereas the later two the DCS ones. Clearly, these two modes can be used to test the s-wave dominance assumption for the meson-pairs in the decays.

\section{Discussions and conclusions}

We have studied the up-down asymmetries in the three-body anti-triplet $\mathbf{B}_{\mathbf{c}} \rightarrow \mathbf{B}_{\mathbf{n}} M M^{\prime}$ decays in the approach of the
$S U(3)_{f}$ symmetry. In our analysis, we have only concentrated on the s-wave $M M^{\prime}$-pair contributions, so that the decays only depend on 12 real irreducible parity conserving and violating amplitudes. With the minimum $\chi^{2}$ fit to the 16 data points, we have obtained a fit with $\chi^{2} /$ d.o. $f=2.4$, which is not relatively good but it will be reduced when more decay branching ratios or up-down asymmetries of the threebody modes are measured in the future. The predictions of the decay branching ratios are slightly different from those in Ref. [25] because the kinematic factor of $\kappa\left(m_{23}^{2}\right)$ highly breaks the $S U(3)_{f}$ flavor symmetry, similar to the cases in the semi-leptonic charmed baryon decays. The triangle relations derived by $[18,25,29]$ still hold since the isospin symmetry preserves in $\kappa\left(m_{23}^{2}\right)$. However, the relations from the U-spin symmetry [30] may be broken by $\kappa\left(m_{23}^{2}\right)$ due to the large mass differences of hadrons. The predicted decay branching ratio of $\mathcal{B}\left(\Lambda_{c}^{+} \rightarrow n \pi^{+} \bar{K}^{0}\right)=(1.1 \pm 0.1) \%$ is 3 times smaller than $(3.6 \pm 0.6) \%$ by the BESIII observation [39]. This indicates that there exist some other sizable contributions to this decay, such as those from $H(\overline{15})$, resonant states and p-wave meson pairs. Our result for the 
ratio of $\frac{\mathcal{B}\left(\Xi_{c}^{+} \rightarrow p K^{-} \pi^{+}\right)}{\mathcal{B}\left(\Xi_{c}^{+} \rightarrow \Xi^{-} \pi^{+} \pi^{+}\right)}=0.50 \pm 0.13$ is 2 times larger than the current experimental value of $0.21 \pm 0.04$. For the averaged up-down asymmetries, both $\pm\langle\alpha\rangle$ are solutions in the $\chi^{2}$ fitting within the $S U(3)_{F}$ approach, which can be determined by experiments. For example, one can measure the angular distribution of the $\Lambda^{0} \pi^{-}$pair in the four-body decay of $\Lambda_{c}^{+} \rightarrow\left(\Xi^{-} \rightarrow \Lambda^{0} \pi^{-}\right) K^{+} \pi^{+}$by BESIII to fix the sign of $\langle\alpha\rangle\left(\Lambda_{c}^{+} \rightarrow \Xi^{-} K^{+} \pi^{+}\right)$, which has been chosen to be negative. We have also examined the decays with the final states involving $K_{L}^{0} / K_{S}^{0}$, which contain the CF and DCS processes. In particular, we have obtained that $\langle\alpha\rangle\left(\Lambda_{c}^{+} \rightarrow \Sigma^{+} K_{S}^{0} K_{L}^{0}\right)=-0.44 \pm 0.32$ and $\langle\alpha\rangle\left(\Xi_{c}^{0} \rightarrow \Xi^{0} K_{S}^{0} K_{L}^{0}\right)=-0.85_{-0.15}^{+0.17}$, which are the same as those for the pure DCS modes of $\Lambda_{c}^{+} \rightarrow \Sigma^{+} K^{0} K^{0}$ and $\Xi_{c}^{0} \rightarrow \Xi^{0} K^{0} K^{0}$, respectively.

Acknowledgements This work was supported in part by National Center for Theoretical Sciences and MoST (MoST-104-2112-M-007-003MY3 and MoST-107-2119-M-007-013-MY3).

Data Availability Statement This manuscript has no associated data or the data will not be deposited. [Authors' comment: It is a theoretical paper so it has no data.]

Open Access This article is distributed under the terms of the Creative Commons Attribution 4.0 International License (http://creativecomm ons.org/licenses/by/4.0/), which permits unrestricted use, distribution, and reproduction in any medium, provided you give appropriate credit to the original author(s) and the source, provide a link to the Creative Commons license, and indicate if changes were made.

Funded by SCOAP ${ }^{3}$.

\section{References}

1. A. Zupanc et al. [Belle Collaboration]. Phys. Rev. Lett. 113, 042002 (2014)

2. M. Ablikim et al., [BESIII Collaboration]. Phys. Rev. Lett. 116, 052001 (2016)

3. M. Tanabashi et al., [Particle Data Group]. Phys. Rev. D 98, 030001 (2018)

4. C.Q. Geng, C.W. Liu, T.H. Tsai, Phys. Lett. B 794, 19 (2019)

5. J.D. Bjorken, Phys. Rev. D 40, 1513 (1989)

6. H.Y. Cheng, B. Tseng, Phys. Rev. D 46, 1042 (1992)
7. H.Y. Cheng, B. Tseng, Phys. Rev. D 55, 1697(E) (1997)

8. H.Y. Cheng, B. Tseng, Phys. Rev. D 48, 4188 (1993)

9. P. Zenczykowski, Phys. Rev. D 50, 402 (1994)

10. Fayyazuddin and Riazuddin, Phys. Rev. D 55, 255

11. Fayyazuddin, Riazuddin, and, Phys. Rev. D 56, 531(E) (1997)

12. R. Dhir, C.S. Kim, Phys. Rev. D 91, 114008 (2015)

13. H.Y. Cheng, X.W. Kang, F. Xu, Phys. Rev. D 97, 074028 (2018)

14. M.J. Savage, R.P. Springer, Phys. Rev. D 42, 1527 (1990)

15. M.J. Savage, Phys. Lett. B 257, 414 (1991)

16. D. Pirtskhalava, P. Uttayarat, Phys. Lett. B 712, 81 (2012)

17. Y. Grossman, D.J. Robinson, JHEP 1304, 067 (2013)

18. C.D. Lu, W. Wang, F.S. Yu, Phys. Rev. D 93, 056008 (2016)

19. C.Q. Geng, Y.K. Hsiao, Y.H. Lin, L.L. Liu, Phys. Lett. B 776, 265 (2017)

20. C.Q. Geng, Y.K. Hsiao, C.W. Liu, T.H. Tsai, JHEP 1711, 147 (2017)

21. D. Wang, P.F. Guo, W.H. Long, F.S. Yu, JHEP 1803, 066 (2018)

22. C.Q. Geng, Y.K. Hsiao, C.W. Liu, T.H. Tsai, Phys. Rev. D 97, $073006(2018)$

23. C.Q. Geng, Y.K. Hsiao, C.W. Liu, T.H. Tsai, Eur. Phys. J. C 78, $593(2018)$

24. C.Q. Geng, C.W. Liu, T.H. Tsai, Phys. Lett. B 790, 225 (2019)

25. C.Q. Geng, Y.K. Hsiao, C.W. Liu, T.H. Tsai, Phys. Rev. D 99, $073003(2019)$

26. Y.K. Hsiao, Y. Yao, H.J. Zhao, Phys. Lett. B 792, 35 (2019)

27. C.Q. Geng, C.W. Liu, T.H. Tsai, S.W. Yeh, Phys. Lett. B 792, 214 (2019)

28. C.P. Jia, D. Wang, F.S. Yu. arXiv:1910.00876 [hep-ph]

29. M. Gronau, J.L. Rosner, C.G. Wohl, Phys. Rev. D 97, 116015 (2018); Addendum: [Phys. Rev. D 98, 073003 (2018)]

30. Y. Grossman, S. Schacht, Phys. Rev. D 99, 033005 (2019)

31. A.J. Buras. arXiv: hep-ph/9806471

32. S. Fajfer, P. Singer, J. Zupan, Eur. Phys. J. C 27, 201 (2003)

33. S. Weinberg, The Quantum Theory of Fields. Vol. 1: Foundations (Cambridge University Press, Cambridge, 1995), pp. 121-134

34. M.S. Sozzi, Discrete Symmetries and CP Violation: From Experiment to Theory. Oxford Graduate Texts (Oxford University Press, New York, 2008), p. 121-185, 231-265

35. M. Berger et al., [Belle Collaboration]. Phys. Rev. D 98, 112006 (2018)

36. Y.B. Li et al., [Belle Collaboration]. Phys. Rev. Lett. 122, 082001 (2019)

37. M. Ablikim et al., [BESIII Collaboration]. Phys. Rev. D 99, 032010 (2019)

38. R. Aaij et al., [LHCb Collaboration]. JHEP 1803, 043 (2018)

39. M. Ablikim et al., [BESIII Collaboration]. Phys. Rev. Lett. 118, 112001 (2017) 